\title{
Update on the worsening particle radiation environment observed by CRaTER and implications for future human deep-space exploration
}

N. A. Schwadron ${ }^{1,2}$, F. Rahmanifard ${ }^{1}$, J. Wilson ${ }^{1,2}$, A. P. Jordan ${ }^{1,2}$, H. E.

Spence $^{1,2}$, C. J. Joyce ${ }^{1}$, J. B. Blake ${ }^{3}$, A. W. Case ${ }^{4}$, W. de Wet ${ }^{5}$, W. M.

Farrell $^{6,2}$, J. C. Kasper ${ }^{7}$, M. D. Looper ${ }^{3}$, N. Lugaz ${ }^{1}$, L. Mays ${ }^{6}$, J. E. Mazur ${ }^{3}$,

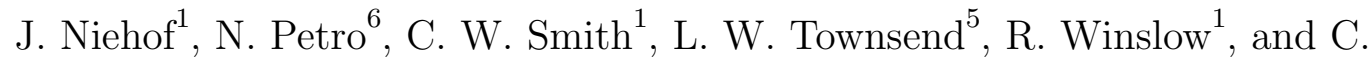

Zeitlin $^{8}$

Corresponding author: N. A. Schwadron, Institute for the Study of Earth, Oceans and Space, Space Science Center, Department of Physics, University of New Hampshire, 8 College Road, Durham NH, 03824 (nschwadron@unh.edu)

${ }^{1}$ University of New Hampshire, Space

This is the author manuscript accepted for publication and has undergone full peer review but has not been through the copyediting, typesetting, pagination and proofreading process, which may lead to differences between this version and the Version of Record. Please cite this article as doi: 10.1002/2017SW001803 
X - 2 SCHWADRON ET AL.: WORSENING GCR RADIATION ENVIRONMENT - UPDATE

${ }_{4}$ Abstract. Over the last decade, the solar wind has exhibited low den-

5 sities and magnetic field strengths, representing anomalous states that have

6 never been observed during the space age. As discussed by Schwadron et al.

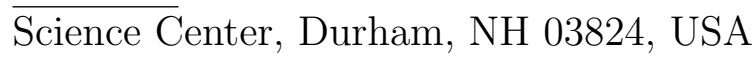

${ }^{2}$ Solar System Exploration Research

Virtual Institute, NASA Ames Research

Center, Moffett Field, CA 94035-0001, USA

${ }^{3}$ The Aerospace Corporation, El Segundo,

CA 90245-4609, USA

${ }^{4}$ High Energy Astrophysics Division,

Harvard Smithsonian Center for

Astrophysics, Cambridge, MA 02138, USA

${ }^{5}$ Dept of Nuclear Engineering, University

of Tennessee, Knoxville, TN, 37996

${ }^{6}$ Goddard Space Flight Center, Greenbelt,

MD 20771, USA

${ }^{7}$ Department of Climate and Space

Science, University of Michigan, Ann Arbor,

MI 48109-2143, USA

${ }^{8}$ Leidos Innovations Corporation,

Houston, TX 77042, USA

February 15, 2018, 10:30pm 
7 (2014a), the cycle 23-24 solar activity led to the longest solar minimum in

s more than 80 years and continued into the "mini" solar maximum of cycle

9 24. During this weak activity, we observed galactic cosmic ray fluxes that

exceeded the levels observed throughout the space age, and we observed small

solar energetic particle events. Here, we provide an update to the Schwadron

et al (2014a) observations from the Cosmic Ray Telescope for the Effects of

Radiation (CRaTER) on the Lunar Reconnaissance Orbiter (LRO). The Schwadron

et al. (2014a) study examined the evolution of the interplanetary magnetic

field, and utilized a previously published study by Goelzer et al. (2013) pro-

jecting out the interplanetary magnetic field strength based on the evolu-

tion of sunspots as a proxy for the rate that the Sun releases coronal mass

ejections (CMEs). This led to a projection of dose rates from galactic cos-

mic rays on the lunar surface, which suggested a $\sim 20 \%$ increase of dose

rates from one solar minimum to the next, and indicated that the radiation

environment in space may be a worsening factor important for consideration

in future planning of human space exploration. We compare the predictions

of Schwadron et al. (2014a) with the actual dose rates observed by CRaTER

in the last 4 years. The observed dose rates exceed the predictions by $\sim 10 \%$,

showing that the radiation environment is worsening more rapidly than pre-

viously estimated. Much of this increase is attributable to relatively low-energy

ions, which can be effectively shielded. Despite the continued paucity of so-

lar activity, one of the hardest solar events in almost a decade occurred in

29 Sept 2017 after more than a year of all-clear periods. These particle radia- 
X - 4 SCHWADRON ET AL.: WORSENING GCR RADIATION ENVIRONMENT - UPDATE

30

31

32

Mars, asteroids and beyond). 


\section{Introduction}

\section{sis} .

(5) ${ }_{38}$ as a function of the effective dose that is related to the energy per unit mass (expressed in $39 \mathrm{~Gy}=\mathrm{joule} / \mathrm{kg}$ ) absorbed by biological tissue, and weighted according to the effectiveness 40 of radiation damage in biological tissue. In this paper, we use recent measurements from ${ }_{41}$ the CRaTER instrument [Cosmic Ray Telescope for the Effects of Radiation, Spence et al., 2010] on the Lunar Reconnaissance Orbiter (LRO) to determine dose rates (data available at http://prediccs.sr.unh.edu/craterweb) .

The deep solar cycle 23-24 minimum and the activity that followed in cycle 24 dif${ }_{45}$ fered significantly from those of the prior solar cycles during the space age [Schwadron et al., 2011; McComas et al., 2013; Schwadron et al., 2014b]. Most recently, Rahmanifard et al. [2017] concluded that we may be entering an era of extremely low solar activity, ${ }_{4}$ such as a Dalton minimum, a Gleissberg minimum or a Maunder minimum. Specifically, Rahmanifard et al. [2017] studied the recent trends in the evolution of the heliospheric magnetic field (HMF) in the context of past solar grand minima, especially the Maunder ${ }_{51}$ period (1645-1715) to gain further insight. A time series of the HMF was reconstructed ${ }_{52}$ from geomagnetic data and measurements from near-Earth spacecraft (OMNI) to find the 53 
$\mathrm{X}-6$ SCHWADRON ET AL.: WORSENING GCR RADIATION ENVIRONMENT - UPDATE

54

ejections (CMEs) into the ambient field, removal of the ambient field through magnetic reconnection, and interchange reconnection between CME and ambient magnetic flux. The minimum value for the HMF at 1 au in the reconstructed magnetic field is $3.13 \pm 0.35$ $\mathrm{nT}$ [Rahmanifard et al., 2017], which is $\sim 1 \mathrm{nT}$ lower than observed in the deep cycle 23-24 minimum. Therefore, the analysis of Rahmanifard et al. [2017] suggests that while we have already observed significant weakening in solar activity, there exists the potential for far weaker activity in coming cycles.

Schwadron et al. [2014a] examined the radiation environment utilizing data from CRaTER and from PREDICCS [Predictions of Radiation from REleASE, EMMREM, and Data Incorporating the CRaTER, COSTEP, and other SEP measurements, http://prediccs.sr.unh.edu Schwadron, 2012]. PREDICCS provides for nowcasting the radiation environment near Earth, at the Moon, and near Mars. Figure 1 shows a key result of Schwadron et al. [2014a] indicating that dose rates (projected to the lunar surface) have grown in the last solar minimum to the highest level observed in the space age. A second major conclusion in Schwadron et al. [2014a] is that solar energetic particle (SEP) events have been quite weak during solar cycle 24. The probability of an SEP event exceeding either the 30-day or 1-year BFO limits is vanishingly small for deep space mission with at least $10 \mathrm{~g} / \mathrm{cm}^{2}$ shielding up to 1 year.

Schwadron et al. [2014a] were able to make a prediction for the evolution of the radiation environment on the lunar surface slightly beyond 2020. The basis of this prediction were the results of Goelzer et al. [2013], in which projections for the interplanetary magnetic field strength were made based on similarity of the solar cycle progression to the Dalton and Gleissberg minima. As seen in Figure 1, both projections showed that galactic cosmic 
SCHWADRON ET AL:: WORSENING GCR RADIATION ENVIRONMENT - UPDATE $\quad \mathrm{X}-7$ conclusions.

\section{Cosmic Ray Telescope for the Effects of Radiation}

The CRaTER instrument consists of a linear stack of 3 pairs of thin and thick silicon detectors, labeled D1 through D6 (Spence et al., 2010, Figure 2). With CRaTER in 
$\mathrm{X}-8$

SCHWADRON ET AL.: WORSENING GCR RADIATION ENVIRONMENT - UPDATE

99

through: 1) $0.81 \mathrm{~mm}\left(0.22 \mathrm{~g} / \mathrm{cm}^{2}\right) \mathrm{Al}$ endcap, 2) D1-D2 detectors, 3) tissue-equivalent plastic (TEP) of thickness $54 \mathrm{~mm}\left(6.09 \mathrm{~g} / \mathrm{cm}^{2}\right)$, 4) D3-D4 detectors, 5) $27 \mathrm{~mm}(3.04$ $\left.\mathrm{g} / \mathrm{cm}^{2}\right)$ TEP, 6) D5-D6 detectors, and 7) $0.81 \mathrm{~mm}\left(0.22 \mathrm{~g} / \mathrm{cm}^{2}\right)$ Al endcap. Further details on CRaTER can be found in Spence et al. (2010). Significant energy loss occurs within the TEP. Therefore, D3-D4 are the most shielded detectors within the instrument.

The energy loss within the TEP allows us to differentiate between particles coming from the Moon and GCRs from deep space at energies below a few hundred $\mathrm{MeV} / \mathrm{nuc}$. As a particle traverses the detector stack, it loses energy, primarily within the TEP. Lowerenergy particles deposit more energy in a detector than higher-energy particles. As a result, a coincident event that is registered in both D4 and D6 (both thick detectors) typically deposits a greater amount of energy in D4 than in D6 if it originates from the direction of the Moon. Conversely, if the particle originates from deep space, it will deposit a greater amount of energy in D6 than in D4, although at high energies, signals in the two detectors are indistinguishable. Note that the coincident rates in D4 and D6 from GCRs are larger than the coincident rates in D2 and D4 due to the larger field-of-view and the comparatively small energy loss within the TEP between D4 and D6 (the piece of TEP between D2 and D4 is thicker than the piece between D4 and D6).

The microdosimeter housed within CRaTER is an early version of what is now a commercially available hybrid that accurately measures total ionizing radiation dose in a silicon target (http://www.teledynemicro.com/product/radiationdosimeter). The CRaTER microdosimeter is behind about $\sim 4.4 \mathrm{~g} / \mathrm{cm}^{2}$ equivalent aluminum, which shields against protons below $\sim 55 \mathrm{MeV}$. Mazur et al. [2011] discussed the first six months of mission data 


\section{Does the Galactic Radiation Hazard Continue to Worsen?}

We test the predictions from Schwadron et al. [2014a] with new CRaTER data from 2014 through 2017 (Fig. 3, bright green). As detailed by Schwadron et al. [2014a], the measurements of ACE in Fig. 3 (red) result from fitting heavy ion distributions measured by ACE/CRIS [Stone et al., 1998] to a model [O'Neill, 2006] for GCR distributions, which 
of GCRs and increases GCR fluxes. The dose rates shown in Figure 1 are therefore higher during the 2008-2009 activity lull compared to the 1997 solar minimum, and the mini-maximum in cycle 24 continues to show relatively weak solar activity.

Goelzer et al. [2013] showed that recent trends are consistent with the beginning of the 1790-1830 period (the Dalton minimum), or the beginning of the 1890-1920 period (the Gleissberg minimum) [Smith et al., 2014]. Solar activity over the next $\sim 5$ years (through 2020) was estimated [Goelzer et al., 2013; Smith et al., 2014] based on the historic behavior in sunspot evolution for the Dalton-like minimum and the Gleissberglike minimum. Recent CRaTER data (bright green points) obtained after the Schwadron et al. [2014a] study are compared to predictions in Figure 3. The CRaTER observations are enhanced relative to the predictions by $\sim 10 \%$, demonstrating an even more rapid increase in radiation dose rates than associated with a Dalton-like or a Gleissberg-like minimum.

Cosmic-ray drifts are known to influence the time-evolution of galactic cosmic ray fluxes [Jokipii et al., 1977]. The cosmic ray drift patterns depend on the quantity $q A$, where $q$ is the cosmic ray charge and the sign of $A$ corresponds to the dominant polarity of the northern heliospheric magnetic field. In cycles with $q A>0$, cosmic rays drift inward near the poles and outward near the heliospheric current sheet. In these cycles, we typically observe "flat-topped" maxima in the time evolution of GCR fluxes. In contrast, cycles with $q A<0$ have cosmic ray drift patterns outward near the poles, and inward near the current sheet causing "peaked" maxima in the time evolution of GCR fluxes [Webber and Lockwood, 1988; Smith, 1990]. For protons and cosmic rays with $q>0$, which contribute most of the GCR dose, the solar minimum between cycles 23 and 24 had $q A<0$ and a 
SCHWADRON ET AL.: WORSENING GCR RADIATION ENVIRONMENT - UPDATE $\quad$ X - 11

peaked maximum in GCR flux, whereas the solar minimum between cycle 24 and 25 will have $q A>0$ and a flat-topped maximum. The more rapid increase in dose rate reported here could be influenced by the change in cosmic ray drift patterns in the transition to the cycle 24-25 solar minimum. For this reason, it will be important to compare the shape of the maximum in dose rates observed by CRaTER in the timeframe of 2020 (the cycle 24-25 GCR flux maximum) with the peaked maximum previously observed in 2009 (the cycle 23-24 GCR flux maximum).

While these observations suggest the evolution toward a grand minimum, it is important to bear in mind that various solar activity models yield disparate predictions, due to a lack of understanding of the underlying mechanisms that drive solar activity. For example, in contrast to the prediction of a prolonged deep minimum, a model based on observations of the solar polar magnetic fields near solar minimum Svalgaard [2017] predicts that the cycle 25 maximum will be stronger than the cycle 24 maximum; this model accurately predicted the weak cycle 24 maximum Svalgaard et al. [2005]. Another well-known model Hathaway and Upton [2016] predicts that the cycle 25 maximum will be about the same as that of cycle 24. Neither of these scenarios is consistent with the onset of a grand minimum.

We also show an update for the sunspot numbers (black curve) in Figure 3 based on the international sunspot number released by Sunspot Index and Longterm Solar Observations (SILSO, http://sidc.oma.be/silso/home). In our 2014 paper we used the original international sunspot number, which has been updated since then to remove the conventional Zurich factor (0.6) and eliminate the effect of a new counting method applied in Zurich by reducing all numbers after 1947 by 18\% 
$\mathrm{X}-12$

SCHWADRON ET AL.: WORSENING GCR RADIATION ENVIRONMENT - UPDATE

189

(http://sidc.oma.be/press/01/welcome.html). Since in our work we use sunspot number (SSN) as a proxy for the solar activity, we are primarily focused on the variations in SSN. In order to be consistent with our previous paper we adapt the new sunspot number data set from SILSO for equivalent sunspot numbers.

Recently, Rahmanifard et al. [2017] investigated the rate of CMEs from LASCO and performed a $\chi$-square analysis to derive the relationship between sunspot number and CME rate. This analysis resulted in lower CME rate than used by Goelzer et al. [2013], and therefore an update to the modulation model developed by Schwadron et al. [2014a].

The minimum dose rate in Figure 3 near the end of 2014 is similar to the minimum dose rate observed, which is $\sim 40 \%$ higher in cycle 24 as compared to cycle 23 . This is important both because of the large increase in dose rate and because the inflection in dose rate is now observed by CRaTER with the addition of new data. The fact that the observed dose rates exceed the model prediction demonstrates that the paucity of solar activity continues to cause elevated cosmic ray fluxes and higher dose rates at this phase of the solar cycle than observed previously in the space age.

Modulation of GCRs by the interplanetary magnetic field is a stochastic process, and the relationship between a given level of activity and the resulting flux in the inner heliosphere is non-trivial. However, broadly speaking, modulation shifts the energy of ions in the local interstellar spectrum (LIS) to lower energies, and depletes the low-energy portion of the spectrum. During periods of weak modulation, fluxes of relatively low-energy ions (with kinetic energies below about $1 \mathrm{GeV} / \mathrm{nuc}$ ) are enhanced compared to periods of strong modulation, but a significant share of these ions have ranges that are insufficient to penetrate moderate depths of shielding. This is particularly true for high-charge ions 
SCHWADRON ET AL:: WORSENING GCR RADIATION ENVIRONMENT - UPDATE $\quad$ X - 13

due to the $\mathrm{Z}^{2}$ dependence of ionization energy loss, and these ions contribute significantly to dose in free space or under thin shielding. Less dramatic increases in dose rates are seen when shielding is more substantial. For example, the NASA OLTARIS model Singleterry et al. [2011] predicts that $30 \mathrm{~g} \mathrm{~cm}^{-2}$ of aluminum shielding produces a roughly $10 \%$ decrease in dose rate for solar minimum conditions, and a roughly $15 \%$ increase under solar maximum conditions, tending to blunt the changes in the incident GCR fluxes.

\section{The Solar Energetic Particle Hazard During Periods of Weak Activity}

Schwadron et al. [2014a] studied the probability of SEP events using PREDICCS [Schwadron, 2012]. They found that the probability of reaching the 30-day Blood Forming Organ (BFO) dose limit of 25 cGy-equivalent behind $10 \mathrm{~g} / \mathrm{cm}^{2}$ aluminum shielding was insignificant over time-scales of 30 days 1 year [NRC, 2008].

While the cycle 24 conditions indicate a low probability for an extreme event, the recent September 102017 SEP event demonstrates that large events can arise with little warning. Figure 4 shows the dose rates in the D1-D2, D3-D4, D5-D6 detectors and CRaTER microdosimeter; the $>1.5$ year lull in activity in 2016-2017 is obvious. The recent September 2017 event was one of the largest of the CRaTER mission, and was rivaled only the March 132012 SEP event in terms of the most shielded D3-D4 and microdosimeter dose rates.

The September 2017 event had an unusually hard spectrum, with large fluxes above 400 $\mathrm{MeV}$, and large dose rates in the most shielded CRaTER detectors. Figure 5 shows the accumulated dose during the event as a function of location and shielding in the CRaTER detector stack accumulated during the event along with the PREDICCS doses modeled through the event into $1 \mathrm{~g} / \mathrm{cm}^{2} \mathrm{H}_{2} \mathrm{O}$, a proxy for a Lens or skin dose. 
In Figure 5, we combined CRaTER observations (yellow triangles) with data from PREDICCS (red points). The blue line and shaded uncertainty region represents a powerlaw fit to the PREDICCS data. The functional form of accumulated dose vs. shielding plotted in Figure 5 allows us to estimate the effective shielding (in $\mathrm{g} / \mathrm{cm}^{2}$ ) for each of the CRaTER detector pairs. The shielding estimates are roughly consistent with the average shielding provided by the TEP, endcaps, and the side-shielding that encircles all six detectors. These effective shielding depths will be useful in estimating the effects of shielding and the attenuation of dose within materials of varying thickness.

These CRaTER shielding estimates for detector pairs are roughly consistent with the shielding associated with the TEP and endcaps. However, radiation penetrates CRaTER from all directions not blocked by the Moon. For example, the endcap provides $0.22 \mathrm{~g} / \mathrm{cm}^{2}$ $\mathrm{Al}$ shielding. However, we find effective D1-D2 shielding of $0.37 \pm 0.02 \mathrm{~g} / \mathrm{cm}^{2}$, which is in excess of the endcap shielding due to extra mass around the detector pair and increased shielding from side-penetrating radiation.

An important question is the degree to which the September, 2017 could have significant health effects for astronauts. We find from Figure 5 that the lens and skin dose on the lunar surface would approach the 30-day limits (100 cGy skin dose and 150 cGy lens dose) [Cucinotta et al., 2010; NRC, 2008; NCRP, 2000]. However, even moderate shielding (>1 $\mathrm{g} / \mathrm{cm}^{2} \mathrm{Al}$ shielding) would reduce the radiation dose below these limits. A question is whether the dose would approach radiation limits during an extravehicular activity (EVA), typically lasting $\sim 3$ hrs. At the peak skin/lens dose rate of $5.8 \pm 0.3 \mathrm{cGy} / \mathrm{hr}$ behind $0.3 \mathrm{~g} / \mathrm{cm}^{2} \mathrm{Al}$ shielding, an astronaut would collect $17.4 \pm 0.9$ cGy dose within $3 \mathrm{hrs}$, which is significantly lower than lens/skin dose limits. This dose and dose rate 
SCHWADRON ET AL:: WORSENING GCR RADIATION ENVIRONMENT - UPDATE $\quad$ X - 15

\section{2}

\section{Successive CMEs in Development of the September 2017 SEP events}


$\mathrm{X}-16$

SCHWADRON ET AL:: WORSENING GCR RADIATION ENVIRONMENT - UPDATE

created a energetic particle seed population that was subsequently accelerated in the September 10 event, similar to twin-CME scenarios studied by Li et al. [2012] and Lugaz et al. [2017].

Both of the X-class flares in September were associated with large and fast interplanetary coronal mass ejections (ICMEs). Figure 7 shows the Wang-Sheeley-Arge (WSA)ENLIL model [Odstrcil et al., 2005] with cone extension for simulating propagation of ICMEs. The simulations were run at the Community Coordinated Modeling Center (CCMC), with the run Leila_Mays_101017_SH_5 and model version number ENLIL 2.8 . The model input parameters for the ICME are as follows: latitude $=-15$ degrees, longitude $=24$ degrees, half-width $=50$ degrees, speed $=1850 \mathrm{~km} / \mathrm{s}$, and a CME leading edge time of 2017-09-06 14:00 UT at the ENLIL inner boundary of 21.5 Rs. For the second ICME the model input parameters are: latitude $=-10$ degrees, longitude $=92$ degrees, halfwidth $=70$ degrees, speed=2800 km/s, and a CME leading edge time of 2017-09-10 17:12 UT at the ENLIL inner boundary of 21.5 Rs. Left panels in Figure 7 show simulated density in the ecliptic plane from 9/6/2017 through 9/8/2017 during the first of the major ICME events. The density enhancement in front of the black contour represents the piled up ICME sheath material. Right panels in Figure 7 show simulated plasma speed in the ecliptic plane. In this case we observe a fast ICME driving a strong compression that presumably forms a shock that sweeps over Earth near 9/8/2017. Note that the modeled fast wind driving the shock that sweeps over Earth has a speed exceeding $900 \mathrm{~km} / \mathrm{s}$.

Figure 8 shows the 1 au plasma signatures observed by ACE at 1 au during the passage of the ICME released after the September 6 X9.3 flare. We show (pink vertical lines) the ICME start time, the ICME Magnetic Ejecta (ME) start time, and the ICME end 
SCHWADRON ET AL.: WORSENING GCR RADIATION ENVIRONMENT - UPDATE $\quad$ X - 17

time. The magnetic field and plasma velocity both show the passage of a shock near the beginning of $9 / 8 / 2017$. The maximum plasma speed is observed slightly in excess of $800 \mathrm{~km} / \mathrm{s}$. At the ME start time to, there is a decrease in magnetic variability, and a clear rotation in the magnetic field. Around the same place where ME starts there is also a decrease in temperature, and the steady decrease in plasma speed. This ICME was directed at Earth, so we observe the ME.

Trailing behind the ICME is a rarefaction region where the density becomes low (> $0.23 \mathrm{~cm}^{-3}$ ) while the magnetic field strength remains close to nominal. As a result, the Alfvén speed becomes large and the Alfvénic Mach number becomes relatively low, $>1.3$. Even lower densities and Alfvénic Mach numbers may be obscured by several data gaps: ACE data gap from 09/09 01:30 to 09/11 00:00 and Wind data gap from 09/10 03:00 to 09/11 00:00. These conditions are reminiscent of density anomaly observed in May, 1999 [Usmanov et al., 2000]. Analysis of this rarefaction region is ongoing.

The WSA-ENLIL simulation of the ICME released after the September 10 flare is shown in Figure 9. The WSA-ENLIL model runs from September 10, 2017 through September 12, 2017 show the propagation of the CME from the Sun to 1 au. This second large ICME was directed much closer to the STEREO-B direction, at longitudes more than $90^{\circ}$ larger than that of Earth. Only a small portion of the modeled sheath of the ICME sweeps past Earth.

The ICME sheath appears well connected to Earth throughout the propagation of the CME from the Sun to $1 \mathrm{au}$. This indicates that the energetic particles accelerated from the CME sheath can propagate to Earth throughout the period in which the ICME propagates to 1 au. The in situ plasma signatures observed at 1 au (Figure 10) also show 
$\mathrm{X}-18$

SCHWADRON ET AL.: WORSENING GCR RADIATION ENVIRONMENT - UPDATE 
SCHWADRON ET AL.: WORSENING GCR RADIATION ENVIRONMENT - UPDATE $\quad$ X - 19

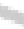

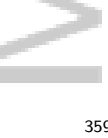

360

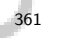


$\mathrm{X}-20$

SCHWADRON ET AL.: WORSENING GCR RADIATION ENVIRONMENT - UPDATE http://prediccs.sr.unh.edu.

\section{References}


SCHWADRON ET AL.: WORSENING GCR RADIATION ENVIRONMENT - UPDATE $\quad$ X - 21

Toroidal Interplanetary Magnetic Field as a Function of Heliolatitude and Solar Cycle, Astrophys. J., 695, 357-362, doi:10.1088/0004-637X/695/1/357.

Cucinotta, F. A., and M. Durante (2006), Cancer rusk from exposure to galactic cosmic rays: implications for space exploration by human beings, J.-Lancet Oncology, 7, 431.

Cucinotta, F. A., S. Hu, N. A. Schwadron, K. Kozarev, L. W. Townsend, and M.-H. Y. Kim (2010), Space radiation risk limits and Earth-Moon-Mars environmental models, Space Weather, 8, S00E09, doi:10.1029/2010SW000572.

Desai, M. I., G. M. Mason, J. R. Dwyer, J. E. Mazur, R. E. Gold, S. M. Krimigis, C. W. Smith, and R. M. Skoug (2003), Evidence for a Suprathermal Seed Population of Heavy Ions Accelerated by Interplanetary Shocks near 1 AU, Astrophys. J., 588, 1149-1162, doi:10.1086/374310.

Desai, M. I., G. M. Mason, J. E. Mazur, and J. R. Dwyer (2006), Solar Cycle Variations in the Composition of the Suprathermal Heavy-Ion Population near 1 AU, Astrophys. J. Lett., 645, L81-L84, doi:10.1086/505935.

Goelzer, M. L., C. W. Smith, N. A. Schwadron, and K. G. McCracken (2013), An analysis of heliospheric magnetic field flux based on sunspot number from 1749 to today and prediction for the coming solar minimum, Journal of Geophysical Research (Space Physics), 118, 7525-7531, doi:10.1002/2013JA019404.

Gopalswamy, N., S. Yashiro, S. Krucker, G. Stenborg, and R. A. Howard (2004), Intensity variation of large solar energetic particle events associated with coronal mass ejections, J. Geophys. Res., 109(A18), 12,105, doi:10.1029/2004JA010602.

Hathaway, D. H., and L. A. Upton (2016), Predicting the amplitude and hemispheric asymmetry of solar cycle 25 with surface flux transport, Journal of Geophysical Research 
(Space Physics), 121(A10), 10, doi:10.1002/2016JA023190.

Hu, S., M.-H. Y. Kim, G. E. McClellan, and F. A. Cucinotta (2009), Modeling the acute health effects of astronauts from exposuite to large solar particle events, Health Physics, 96, 465 - 476, doi:10.1097/01.HP.0000339020.92837.61.

Jokipii, J. R., E. H. Levy, and W. B. Hubbard (1977), Effects of particle drift on cosmicray transport. i - general properties, application to solar modulation, Astrophys. J., 213, 861-868, doi:10.1086/155218.

Li, G., R. Moore, R. A. Mewaldt, L. Zhao, and A. W. Labrador (2012), A Twin-CME Scenario for Ground Level Enhancement Events, Space Sci. Rev., 171, 141-160, doi: 10.1007/s11214-011-9823-7.

Lugaz, N., M. Temmer, Y. Wang, and C. J. Farrugia (2017), The Interaction of Successive Coronal Mass Ejections: A Review, Solar Phys., 292, 64, doi:10.1007/s11207-017-10916.

Mazur, J. E., W. R. Crain, M. D. Looper, D. J. Mabry, J. B. Blake, A. W. Case, M. J. Golightly, J. C. Kasper, and H. E. Spence (2011), New measurements of total ionizing dose in the lunar environment, Space Weather, 9, S07002, doi:10.1029/2010SW000641. Mazur, J. E., C. Zeitlin, N. Schwadron, M. D. Looper, L. W. Townsend, J. B. Blake, and H. Spence (2015), Update on Radiation Dose From Galactic and Solar Protons at the Moon Using the LRO/CRaTER Microdosimeter, Space Weather, 13, 363-364, doi:10.1002/2015SW001175.

McComas, D. J., N. Angold, H. A. Elliott, G. Livadiotis, N. A. Schwadron, R. M. Skoug, and C. W. Smith (2013), Weakest solar wind of the space age and the current "Mini" solar maximum, The Astrophysical Journal, 779, 2, doi:10.1088/0004-637X/779/1/2. 
SCHWADRON ET AL.: WORSENING GCR RADIATION ENVIRONMENT - UPDATE $\quad$ X - 23

NCRP (2000), Radiation protection guidance for activities in low-Earth orbit, NCRP Report 132, National Council on Radiation Protection and Measurements, Bethesda (MD).

NRC (2008), Managing Space Radiation Risk in the New Era of Space Exploration, National Academy Press, The National Academies Press, Washington, DC.

Odstrcil, D., V. J. Pizzo, and C. N. Arge (2005), Propagation of the 12 May 1997 interplanetary coronal mass ejection in evolving solar wind structures, Journal of Geophysical Research (Space Physics), 110, A02106, doi:10.1029/2004JA010745.

O’Neill, P. M. (2006), Badhwar O’Neill galactic cosmic ray model update based on advanced composition explorer (ACE) energy spectra from 1997 to present, Advances in Space Research, 37, 1727-1733, doi:10.1016/j.asr.2005.02.001.

Rahmanifard, F., N. A. Schwadron, C. W. Smith, K. G. McCracken, K. A. Duderstadt, N. Lugaz, and M. L. Goelzer (2017), Inferring the Heliospheric Magnetic Field Back through Maunder Minimum, Astrophys. J., 837, 165, doi:10.3847/1538-4357/aa6191.

Schwadron, N. (2012), Near-real-time situational awareness of space radiation hazards, Space Weather, 10, 10,005, doi:10.1029/2012SW000860.

Schwadron, N. A., L. A. Fisk, and G. Gloeckler (1996), Statistical acceleration of interstellar pick-up ions in co-rotating interaction regions, GRL, 23, 2871-2874, doi: 10.1029/96GL02833.

Schwadron, N. A., C. W. Smith, H. E. Spence, J. C. Kasper, K. Korreck, M. L. Stevens, B. A. Maruca, K. K. Kiefer, S. T. Lepri, and D. McComas (2011), Coronal electron temperature from the solar wind scaling law throughout the space age, The Astrophysical Journal, 739, 9, doi:10.1088/0004-637X/739/1/9. 
$\mathrm{X}-24$ SCHWADRON ET AL.: WORSENING GCR RADIATION ENVIRONMENT - UPDATE

Schwadron, N. A., T. Baker, B. Blake, A. W. Case, J. F. Cooper, M. Golightly, A. Jordan, C. Joyce, J. Kasper, K. Kozarev, J. Mislinski, J. Mazur, A. Posner, O. Rother, S. Smith, H. E. Spence, L. W. Townsend, J. Wilson, and C. Zeitlin (2012), Lunar radiation environment and space weathering from the cosmic ray telescope for the effects of radiation (CRaTER), Journal of Geophysical Research (Planets), 117, doi: 10.1029/2011JE003978.

Schwadron, N. A., J. B. Blake, A. W. Case, C. J. Joyce, J. Kasper, J. Mazur, N. Petro, M. Quinn, J. A. Porter, C. W. Smith, S. Smith, H. E. Spence, L. W. Townsend, R. Turner, J. K. Wilson, and C. Zeitlin (2014a), Does the worsening galactic cosmic radiation environment observed by CRaTER preclude future manned deep space exploration?, Space Weather, 12, 622-632, doi:10.1002/2014SW001084.

Schwadron, N. A., M. L. Goelzer, C. W. Smith, J. C. Kasper, K. Korreck, R. J. Leamon, S. T. Lepri, B. A. Maruca, D. McComas, and M. L. Steven (2014b), Coronal electron temperature in the protracted solar minimum, the cycle 24 mini maximum, and over centuries, Journal of Geophysical Research: Space Physics, doi:10.1002/2013JA019397.

Schwadron, N. A., M. A. Lee, M. Gorby, N. Lugaz, H. E. Spence, M. Desai, T. Török, C. Downs, J. Linker, R. Lionello, Z. Mikić, P. Riley, J. Giacalone, J. R. Jokipii, J. Kota, and K. Kozarev (2015), Particle Acceleration at Low Coronal Compression Regions and Shocks, Astrophys. J., 810, 97, doi:10.1088/0004-637X/810/2/97.

Singleterry, R. C., Jr., S. R. Blattnig, M. S. Clowdsley, G. D. Qualls, C. A. Sandridge, L. C. Simonsen, T. C. Slaba, S. A. Walker, F. F. Badavi, J. L. Spangler, A. R. Aumann, E. N. Zapp, R. D. Rutledge, K. T. Lee, R. B. Norman, and J. W. Norbury (2011), OLTARIS: On-line tool for the assessment of radiation in space, Acta Astronautica, 68, 
SCHWADRON ET AL.: WORSENING GCR RADIATION ENVIRONMENT - UPDATE $\quad \mathrm{X}-25$ 


\section{5-3768, doi:10.1029/2000GL000082.}

Webber, W. R., and J. A. Lockwood (1988), Characteristics of the 22-year modulation of cosmic rays as seen by neutron monitors, J. Geophys. Res., 93, 8735-8740, doi: 10.1029/JA093iA08p08735. 
Figure 1. Evolving and increasingly hazardous radiation levels in space. Top Panel: ACE dose rates (red) are based on fits to CRIS spectra [O'Neill, 2006], CRaTER measurements (green) from the zenith facing D1/D2 detectors are used as proxies for lens dose rates behind $0.3 \mathrm{~g} / \mathrm{cm}^{2} \mathrm{Al}$ shielding Schwadron et al. [2012]. The sunspot number predictions (the lower blue and red dashed lines) show two cases based on a Gleissberglike and a Dalton-like minimum, the results of which are similar. The dose predictions (solid black curve and the upper blue and red curves) are from a sunspot-based model of the heliospheric magnetic field and the correlated variation in modulation of GCRs [Appendix A Schwadron et al., 2014a]. The ACE data, CRaTER data, and model results are projected to the lunar surface. Bottom Panel: Same as top panel but for a longer time span. [From Schwadron et al., 2014a].

Figure 2. The CRaTER instrument consists of a stack of 3-pairs of thin and thick silicon detectors separated by tissue-equivalent plastic (TEP). Shown here is the configuration of these detectors with D1-D2 facing zenith, and D5-D6 facing in the nadir direction. Note that the D3-D4 detectors are the most shielded thin-thick pair within the instrument. 
Figure 3. Recent CRaTER data (bright green) are updated after the Schwadron et al. [2014a] study to further test the predictions. The sunspot number predictions from Goelzer et al. [2013] (the lower blue and red curves) show two cases based on a Gleissberg-like and a Dalton-like minimum, the results of which are similar. Updates to the sunspot number (lower black curve) are adapted from the international sunspot number released by Sunspot Index and Long-term Solar Observations (SILSO, http://sidc.oma.be/silso/home). The dose predictions (solid black curve and the upper red and blue curves) are from a sunspot-based model of the heliospheric magnetic field and the correlated variation in modulation of GCRs [Appendix A Schwadron et al., 2014a]. The ACE data, CRaTER data, and model results are projected geometrically to the lunar surface.

Figure 4. Dose rates in the three thin-thick detector pairs (D1-D2, D3-D4, D5-D6) and the microdosimeter within CRaTER. All dose rates have been geometrically corrected for exposure on the lunar surface and corrected for doses in $\mathrm{H}_{2} \mathrm{O}$ as opposed to $\mathrm{Si}$ [Schwadron et al., 2012]. 
Figure 5. Accumulated doses on the lunar surface during the September 2017 SEP event behind different amounts of $\mathrm{Al}$ shielding. Red data points show PREDICCS data including uncertainties. The blue line and shaded uncertainty region shows power-law fit to the PREDICCS data, $D=D_{0}\left(s / s_{0}\right)^{\gamma}$, where $D$ is dose, $s$ is Al shielding thickness, and $s_{0}=1 \mathrm{~g} / \mathrm{cm}^{2}$. The fits have the following coefficients: (top) $D_{0}=35.91 \pm 5.45$ cGy, $\gamma=-0.90 \pm 0.10$, (bottom) $D_{0}=3.56 \pm 0.14 \mathrm{cGy}, \gamma=-0.11 \pm 0.03$. In the top panel, we find the accumulated doses for D1-D2, D3-D4, and D5-D6 and the intersection with the power-law fit to estimate the effective shielding for each of these CRaTER thin-thick detector pairs. The numbers and uncertainties next to thin-thick detector pairs indicate effective $\mathrm{Al}$ shielding depth (in $\mathrm{g} / \mathrm{cm}^{2}$ ). In the bottom panel, we show PREDICCS data and the power-law fit for doses in $10 \mathrm{~g} / \mathrm{cm}^{2} \mathrm{H}_{2} \mathrm{O}$. Note that CRaTER detector pair dose rates are comparable to doses in the Lens and Skin $(\sim 1$ $\mathrm{g} / \mathrm{cm}^{2}$ of $\mathrm{H}_{2} \mathrm{O}$ as a proxy). However, the TEP between D1-D2 and D3-D4 is $\sim 6.09$ $\mathrm{g} / \mathrm{cm}^{2}$ and therefore not sufficiently thick to evaluate the large internal mass $\left(\sim 10 \mathrm{~g} / \mathrm{cm}^{2}\right.$ of $\mathrm{H}_{2} \mathrm{O}$ as a proxy) associated with Organ and BFO doses. Therefore, the lower panel includes only PREDICCS data and the power-law fit, but does not contain CRaTER data. 
Figure 6. Large X-class flares began each of the major events observed in September 2017. The top panels include observations of the erupting active region observed by the Solar Dynamics Observatory (SDO), courtesy of NASA/SDO and the AIA, EVE, and HMI science teams. The left top solar image of the September 6 X9.3 flare (11:58 UT) is from telescope AIA 131. The top right image of the September 10 X8.2 flare (16:06) is a combination of wavelengths that includes AIA 193. Dose rates on the lunar surface from CRaTER (panel 2) and PREDICCS are shown throughout both events. Note that dose rates in free space are approximately $2 \times$ those on the lunar surface. In the bottom panel, we show energetic particle differential fluxes from GOES.

Figure 7. Propagation ICMEs from September 6 through September 8, 2017 based on WSA-ENLIL simulations. These CMEs followed the X9.3 flare event on September 6 . The ICMEs over this period propagate toward Earth, and a strong compression region swept past Earth on September 7. These runs were performed by the CCMC.

Figure 8. In situ plasma signatures of the ICME and Magnetic Ejecta (ME) observed on September 7 and 8. Pink vertical lines indicate the ICME start time, the ICME ME start time, and the ICME end time. The panels (top to bottom) correspond to: the solar wind magnetic field strength and RTN components, density, speed, temperature, plasma beta, and Alfvén mach number. 
Figure 9. Propagation of ICMEs from September 10 (16:00 UT) through September 12 (16:00 UT), 2017 based on WSA-ENLIL simulations. These ICMEs followed the X8.2 flare event on September 10. The ICMEs over this period propagate toward the STEREOB direction, at longitudes more than $90^{\circ}$ larger than that of Earth. These runs were performed by the CCMC.

Figure 10. In situ plasma signatures of the ICME and Magnetic Ejecta (ME) observed on September 12 and 13. Pink vertical lines indicate the ICME start time, the ICME ME start time, and the ICME end time. The panels (top to bottom) correspond to: the solar wind magnetic field strength and RTN components, density, speed, temperature, plasma beta, and Alfvén mach number.

Figure 11. Summary of observations of the September 6 and September 10 SEP events. We note that the September 6 event resulted in a CME directed toward Earth, whereas the September $10 \mathrm{CME}$ was directed $90^{\circ}$ longitudinally forward. As a result, the first Earth directed event showed the passage of the CME, the associated shock and the energetic storm particles (ESP) accelerated in interplanetary space. In contrast, the September 10 SEP event did not show a pronounced shock-associated ESP enhancement in energetic particles. 
Prediction (Dalton)

Prediction (Gleissberg)

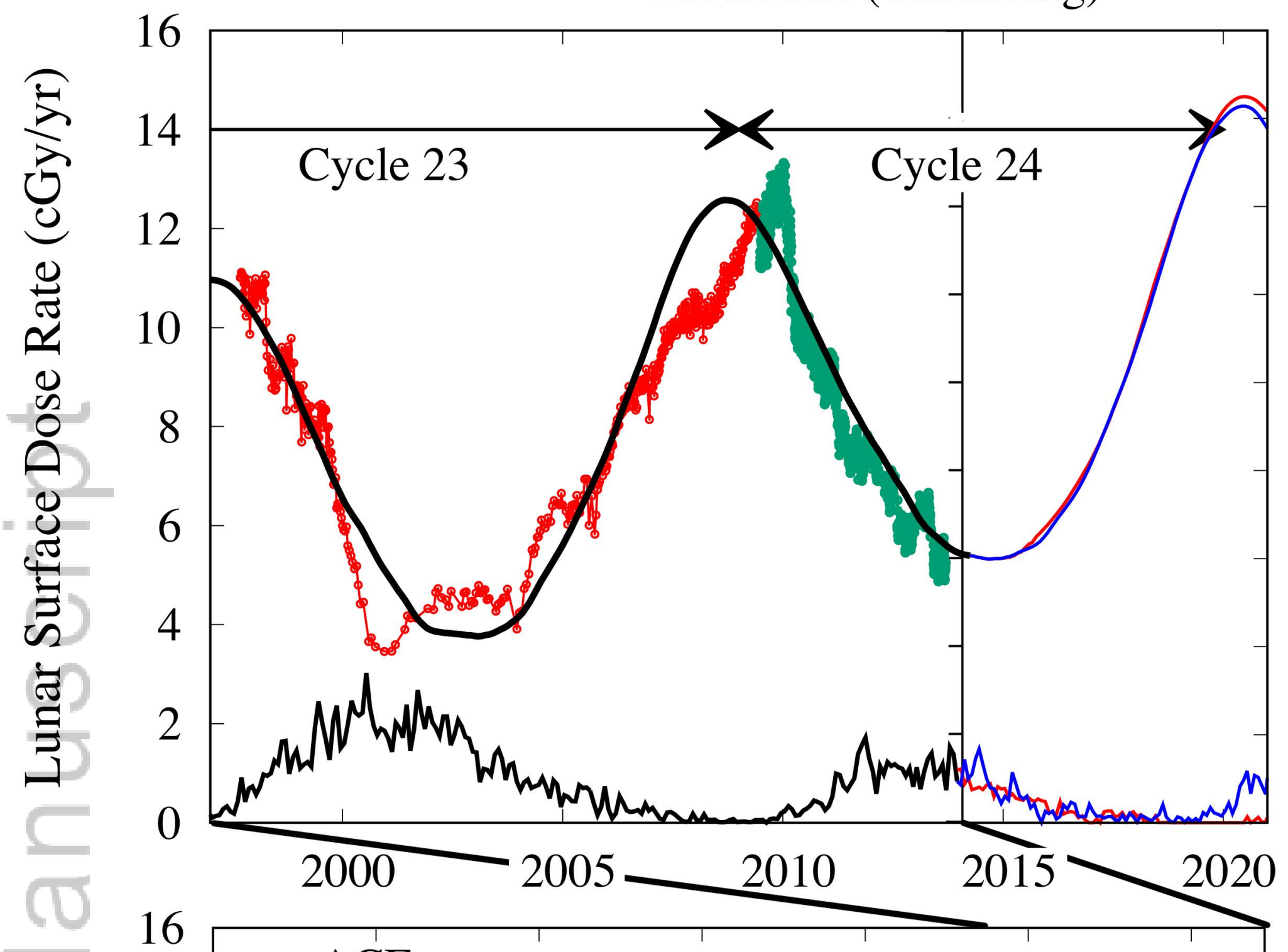

300

200

100

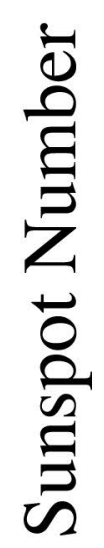

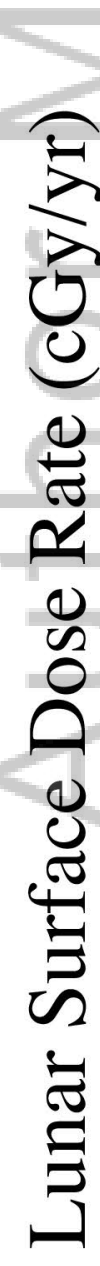

16

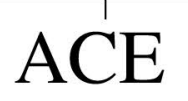

14 CRaTER

12

10 


\section{DEEP-SPACE (ZENITH) SHIELD}

D1 - 148 um
D2 - $1 \mathrm{~mm}$

$54.0 \mathrm{~mm}$
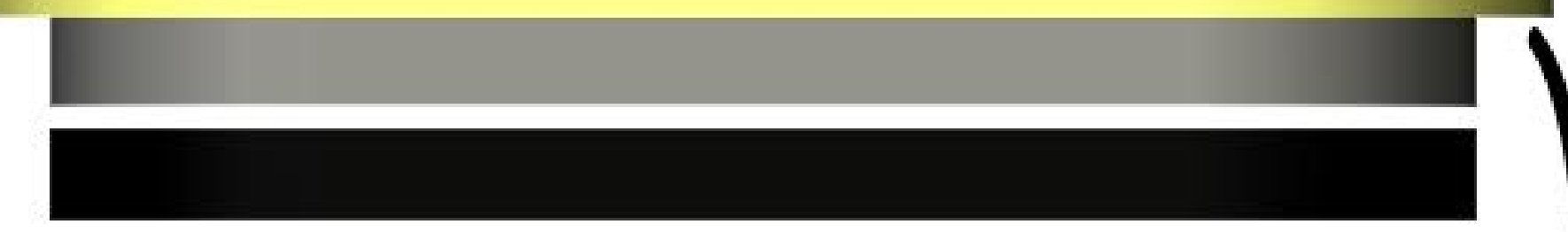

D3 - 149 um

D4 - $1 \mathrm{~mm}$

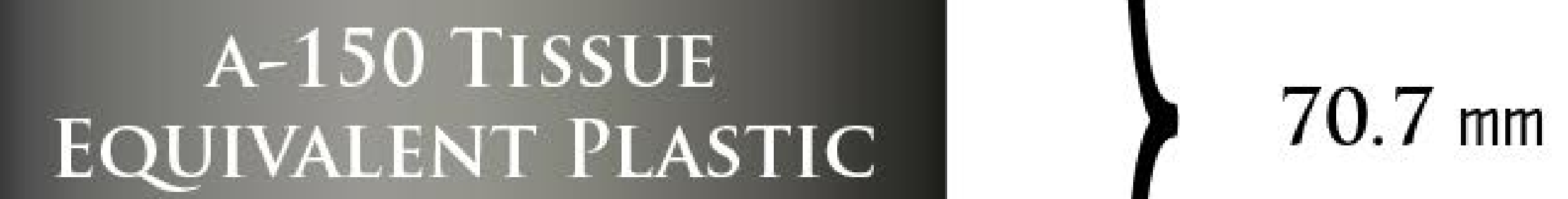

D5 - 149 um

D6 - $1 \mathrm{~mm}$

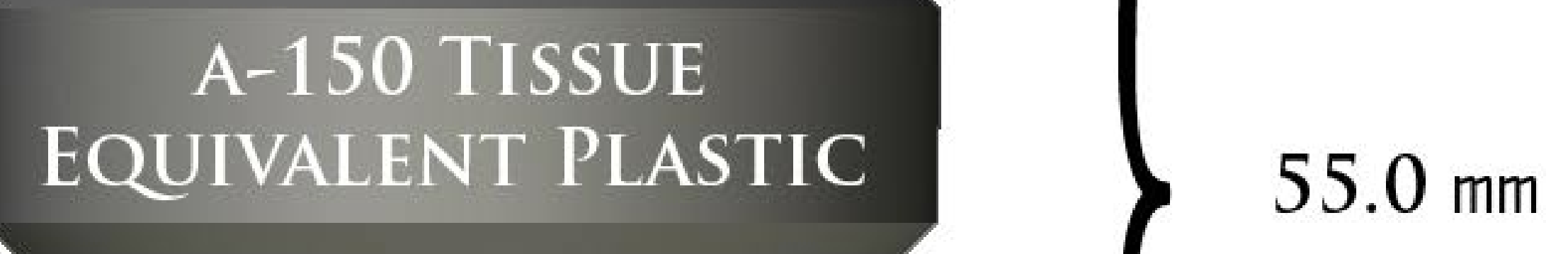

\section{LUNAR (NADIR) SHIELD}




\section{Prediction (Dalton)}

Prediction (Gleissberg)
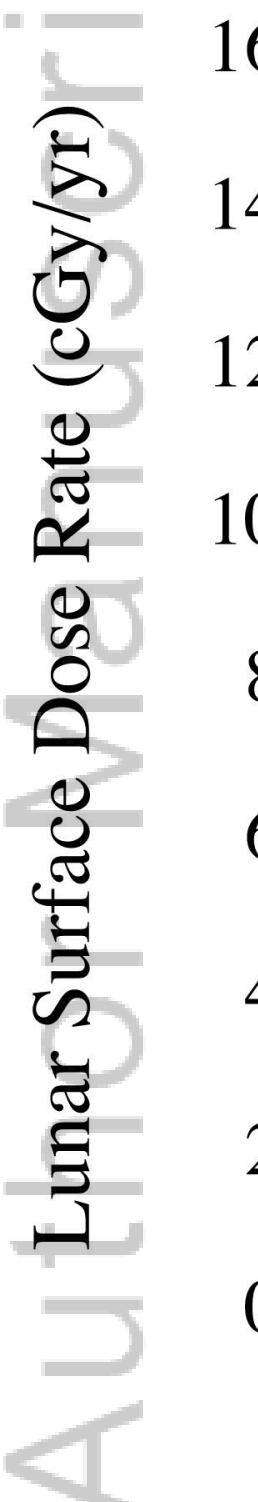

16
14
12
10
8
6
4
2
0
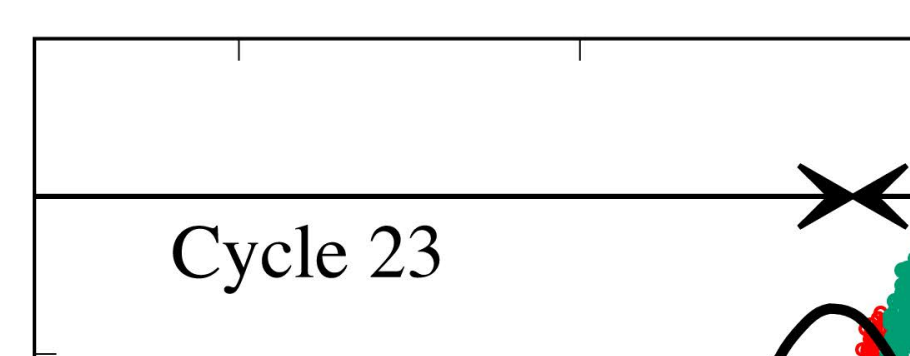


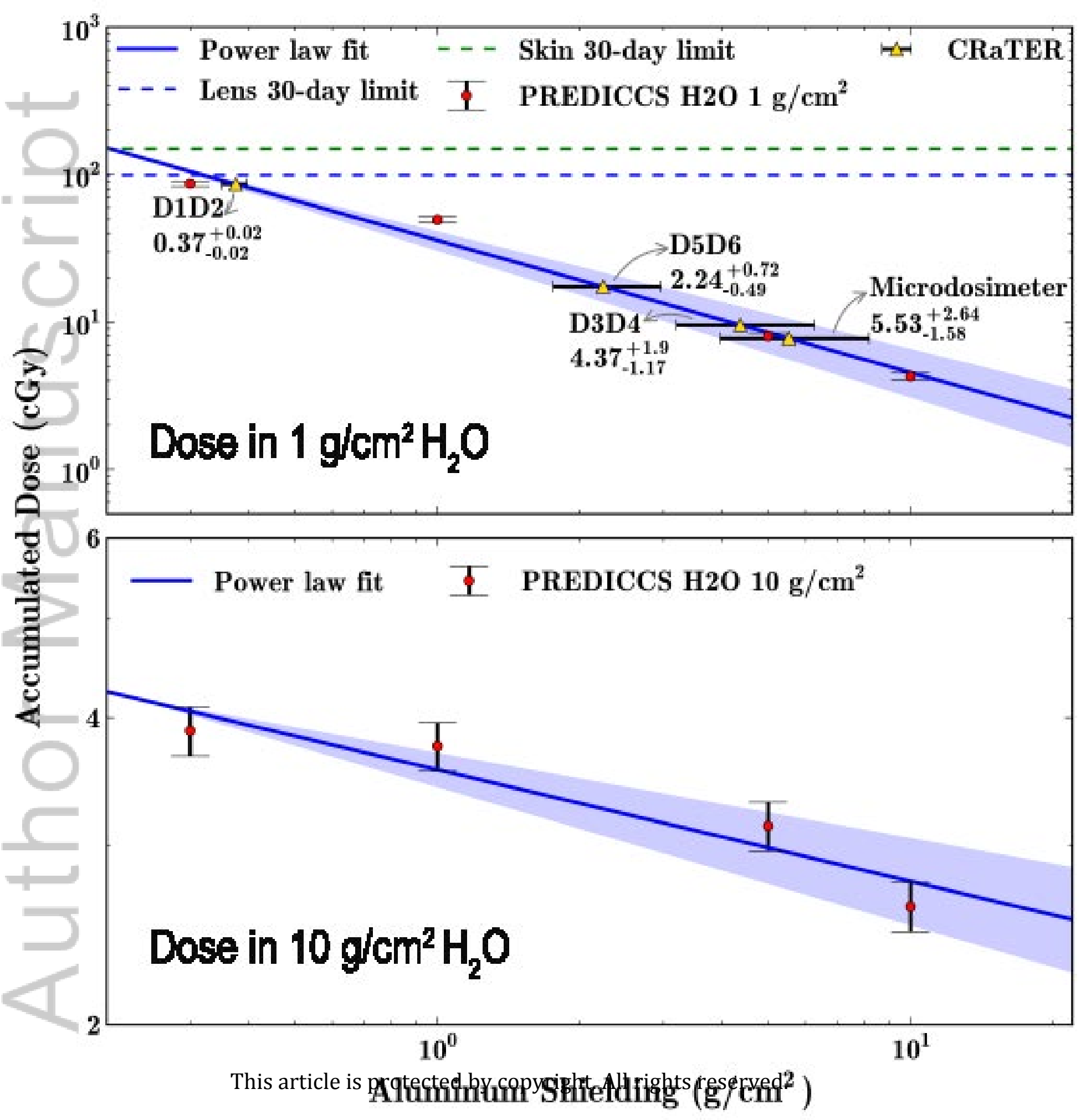


X9.3 Flare

Sep 6

\section{X8.2 Flare}

Sep 10
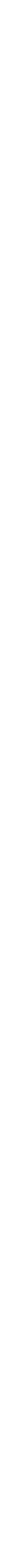

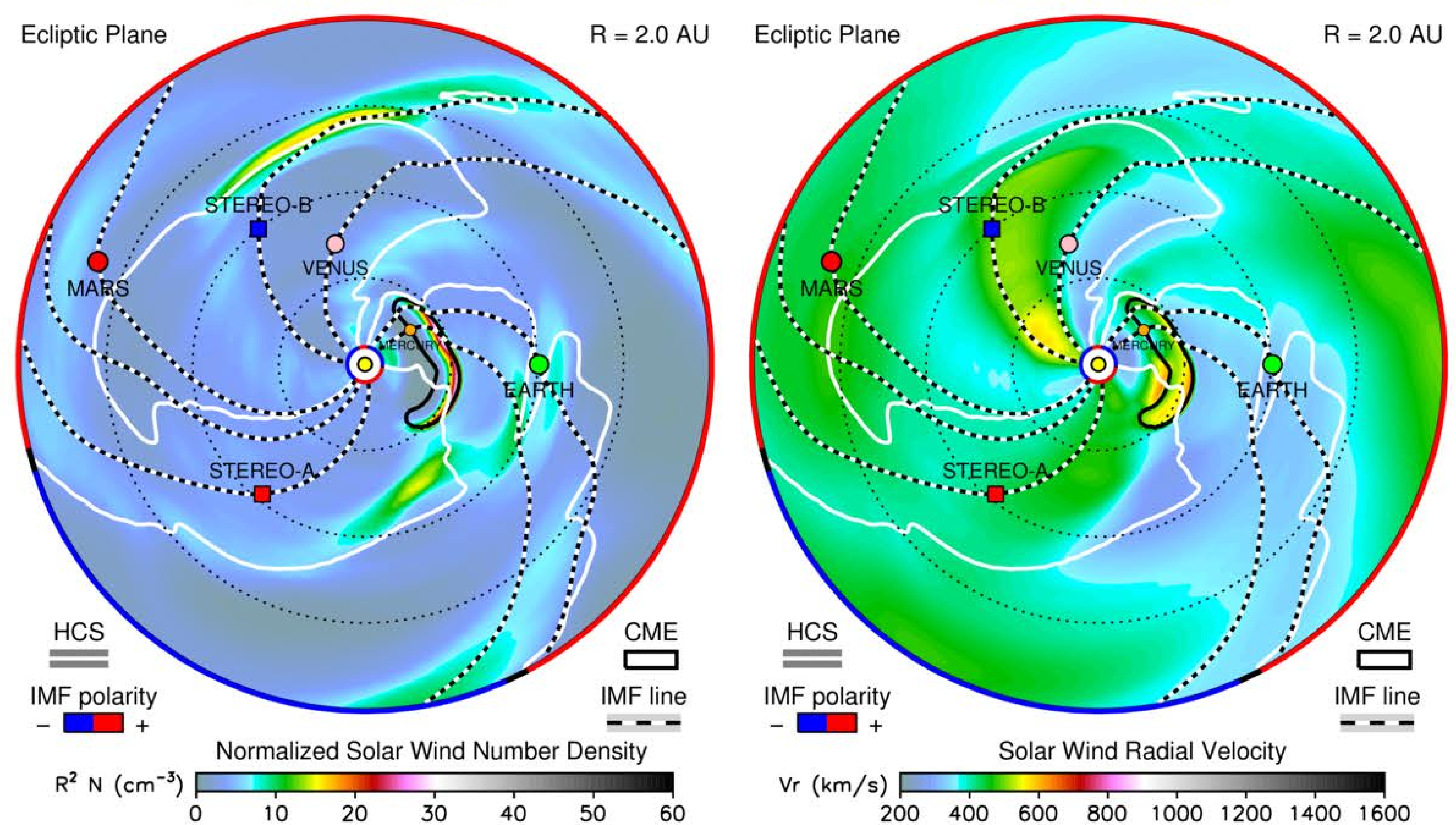

$\operatorname{Vr}(\mathrm{km} / \mathrm{s})$

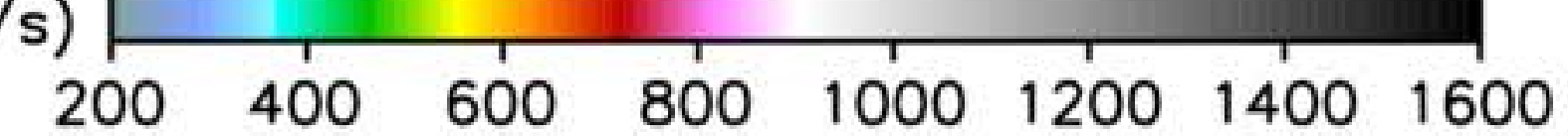

ENLIL-lowres + a6b1 GONGb-WSAdt+Cone- CCMC HelioWeather @ CCMC ENLIL-lowres + a6b1 GONGb-WSAdt+Cone-CCMC HelioWeather @ CCMC

\section{7-09-07T00}

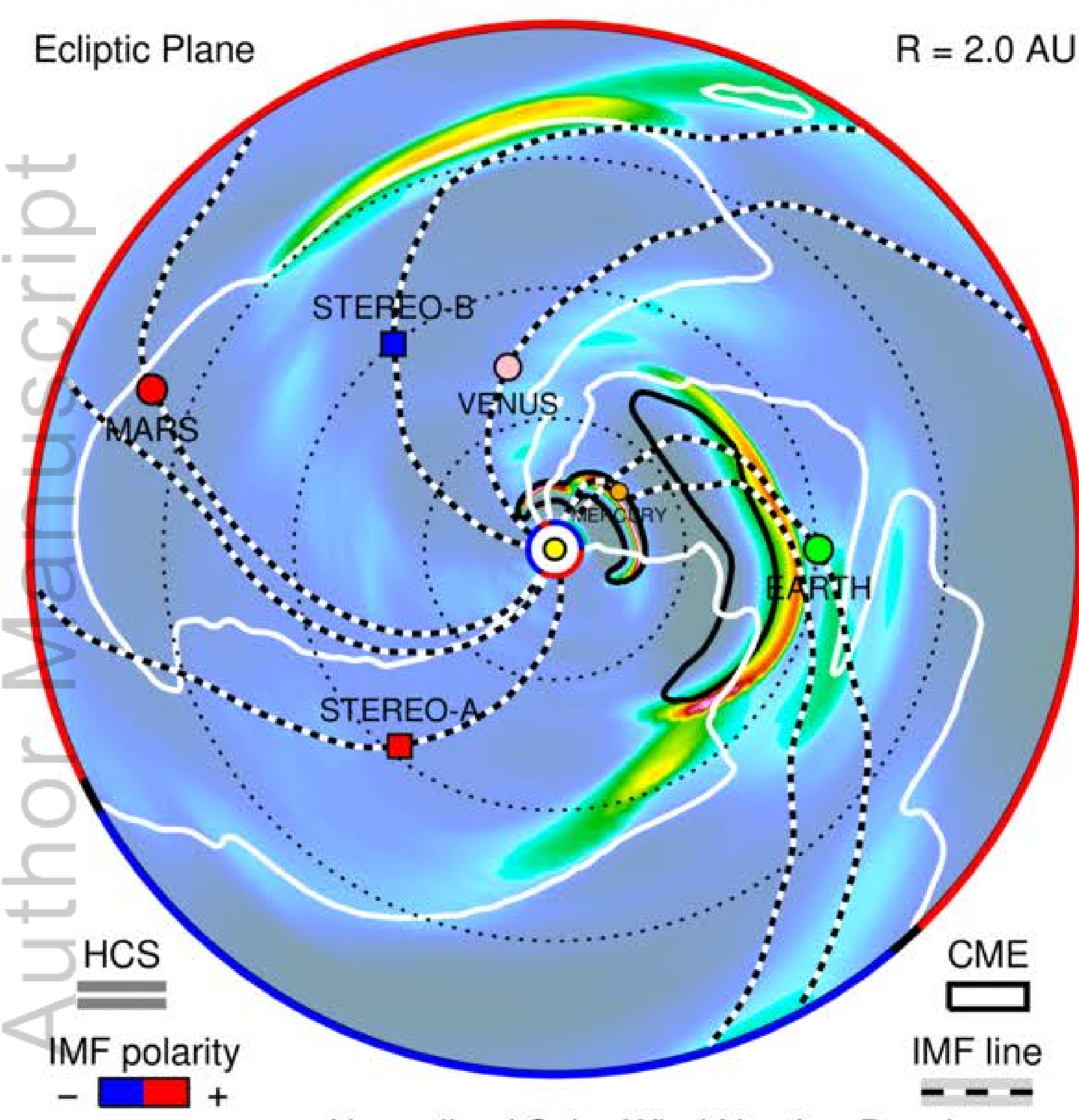

Normalized Solar Wind Number Density
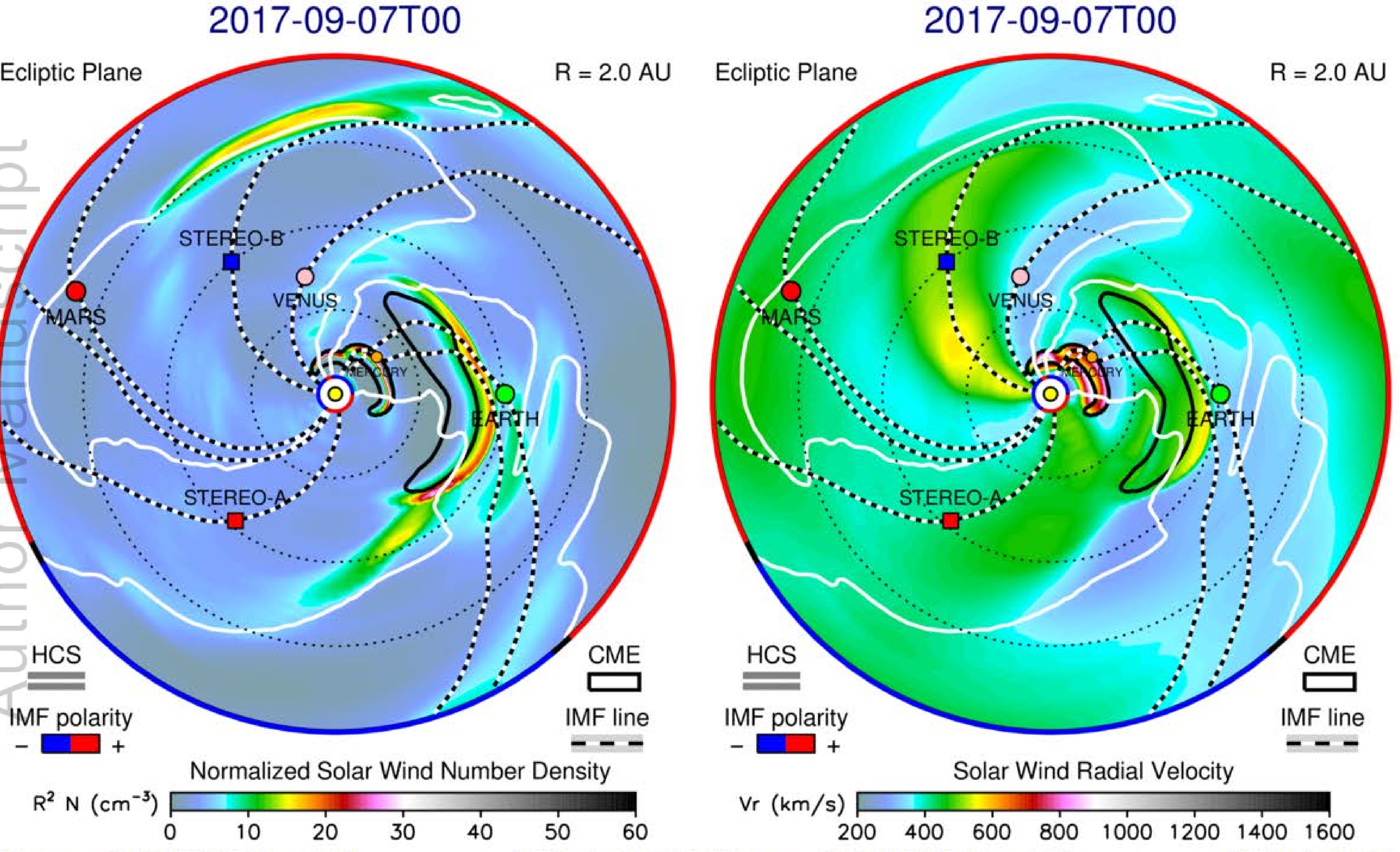

ENLIL-lowres + a6b1 GONGb-WSAdt+Cone - CCMC

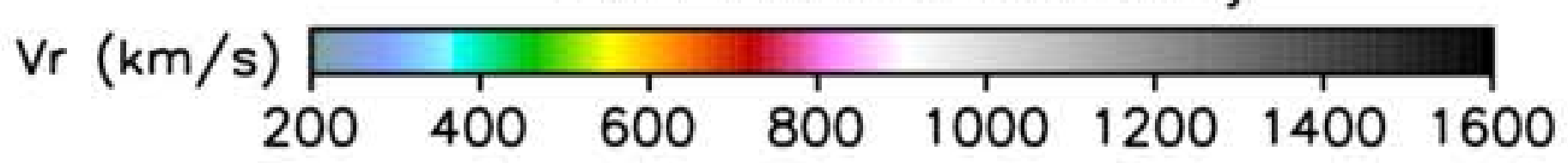

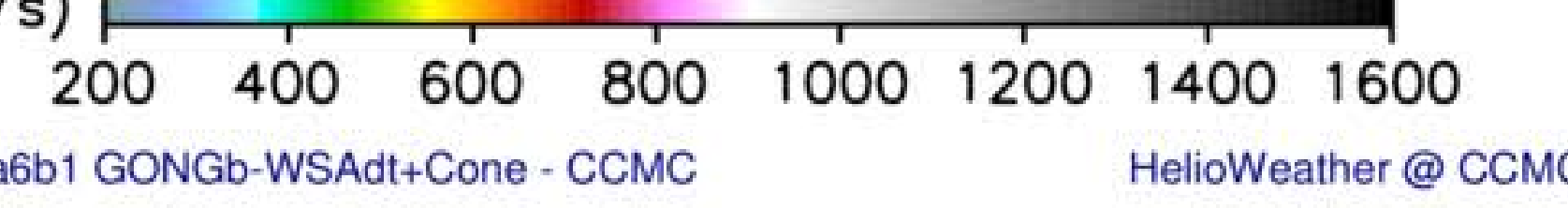

2017-09-08T00

2017-09-08T00

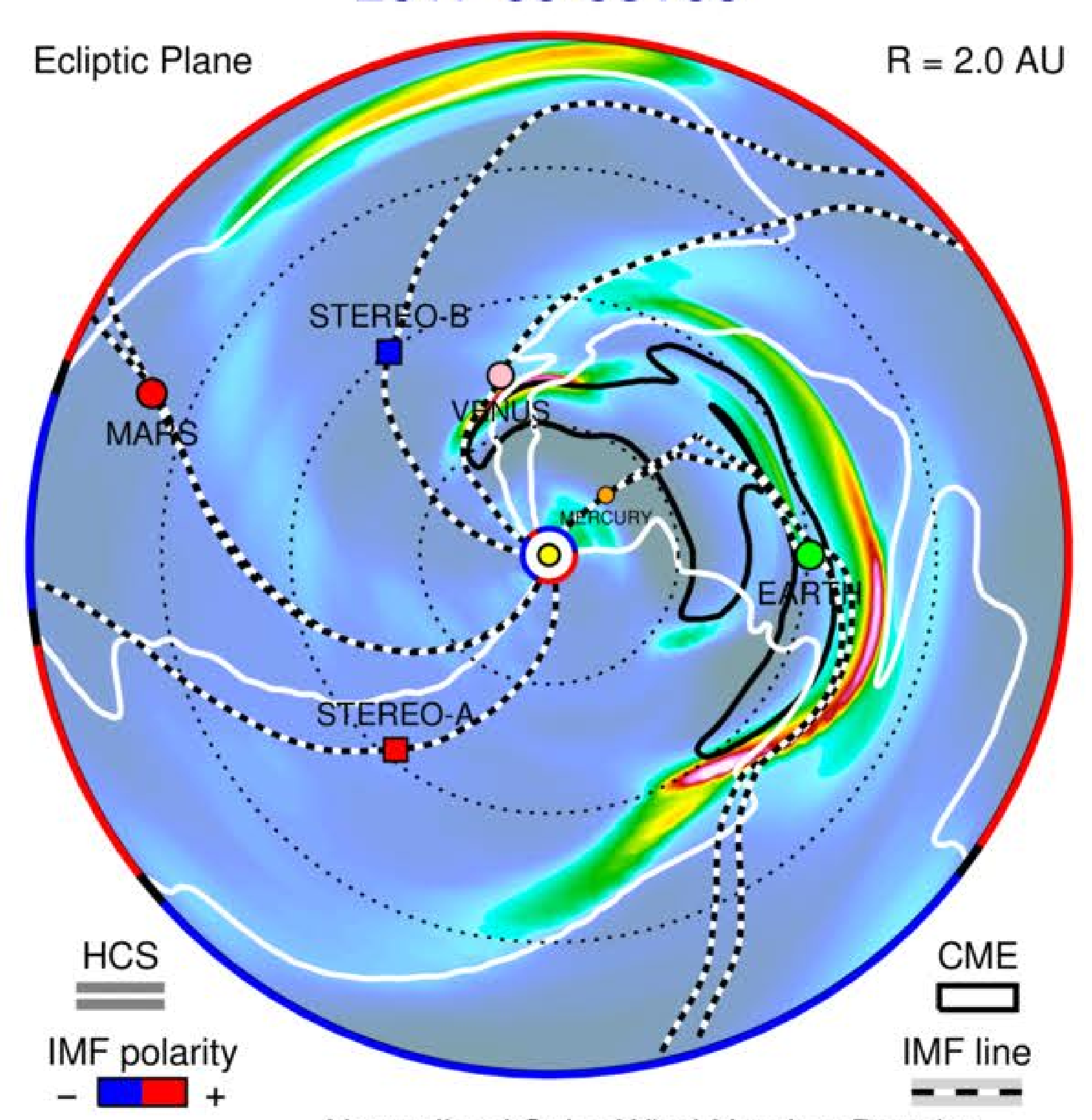

Normalized Solar Wind Number Density
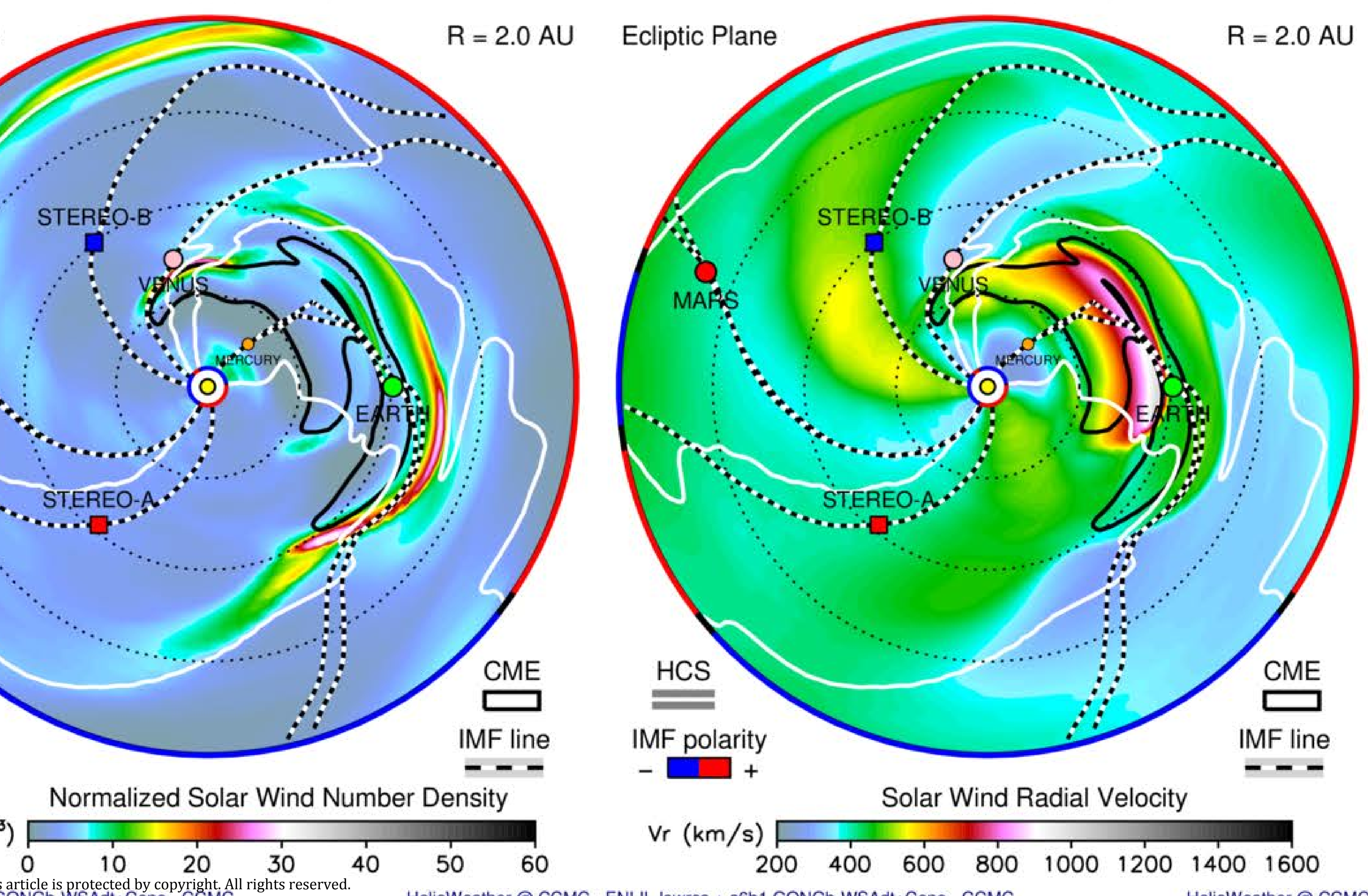

$\operatorname{Vr}(\mathrm{km} / \mathrm{s})$ Solar Wind Radial Velocity

$\begin{array}{llllllll}200 & 400 & 600 & 800 & 1000 & 1200 & 1400 & 1600\end{array}$

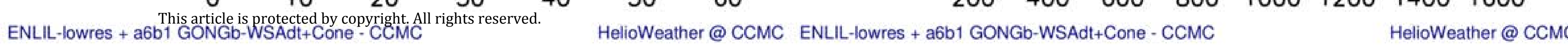



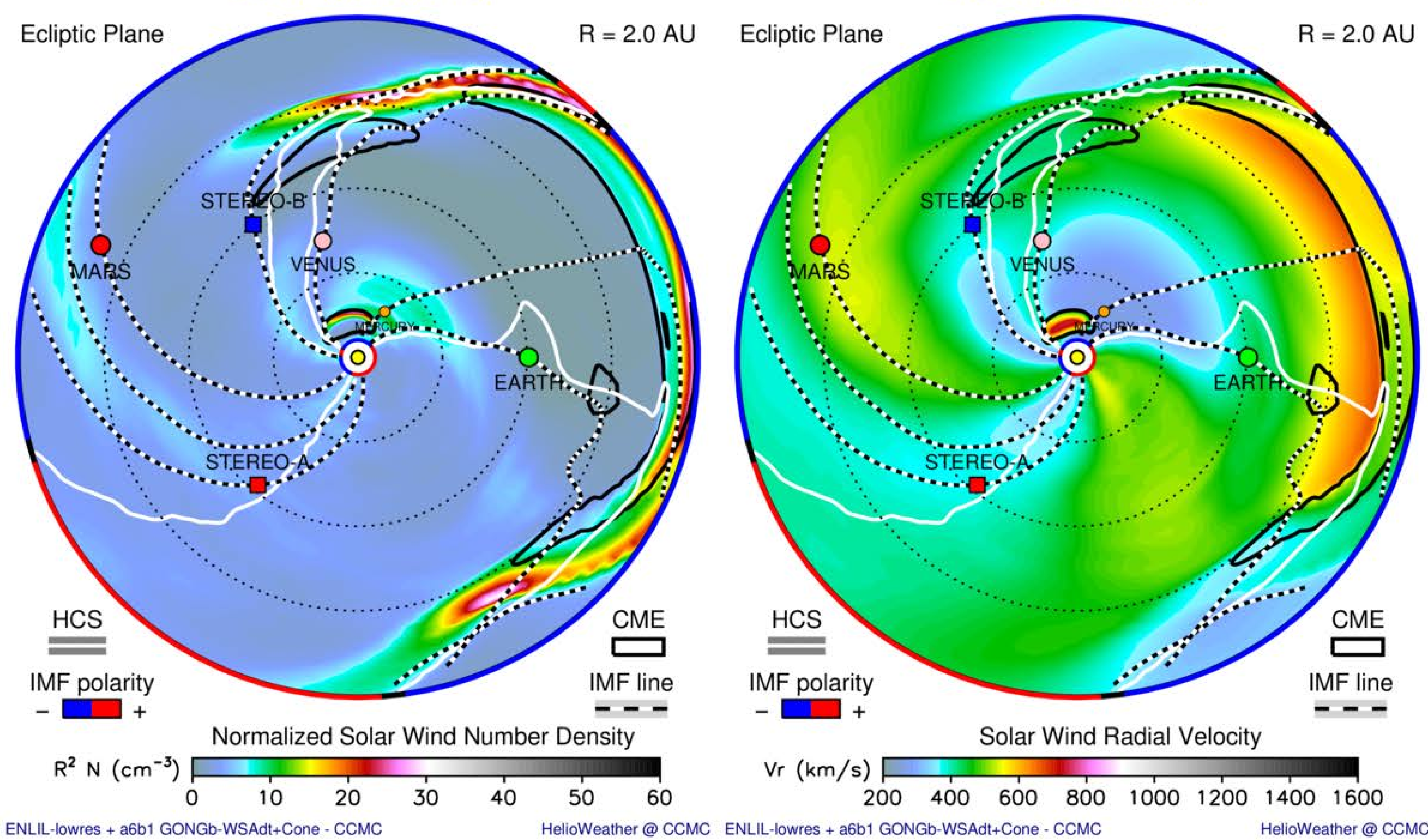

$\operatorname{Vr}(\mathrm{km} / \mathrm{s})$

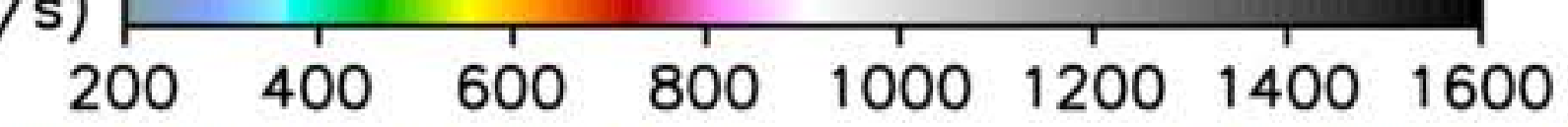

\section{7-09-11T16}

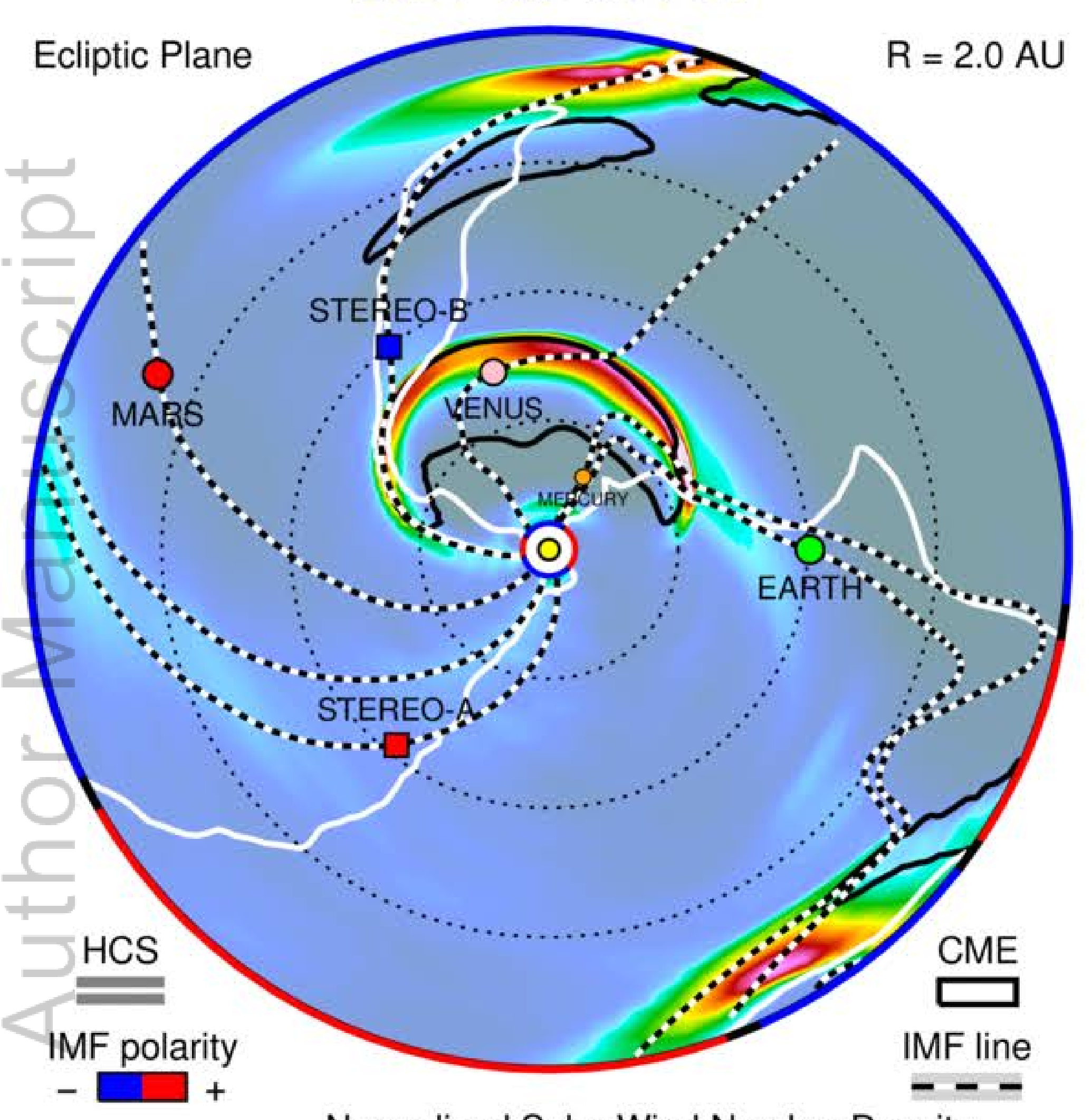

Normalized Solar Wind Number Density

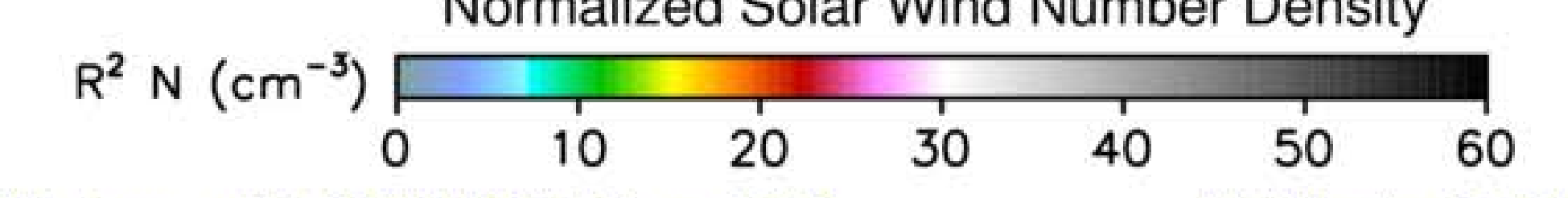

ENLIL-lowres + a6b1 GONGb-WSAdt+Cone- CCMC HelioWeather @ CCMC

ENLIL-lowres + a6b1 GONGb-WSAdt+Cone-CCMC HelioWeather@ CCMC ENLIL-lowres + a6b1 GONGb-WSAdt+Cone-CCMC HelioWeather@ CCMC

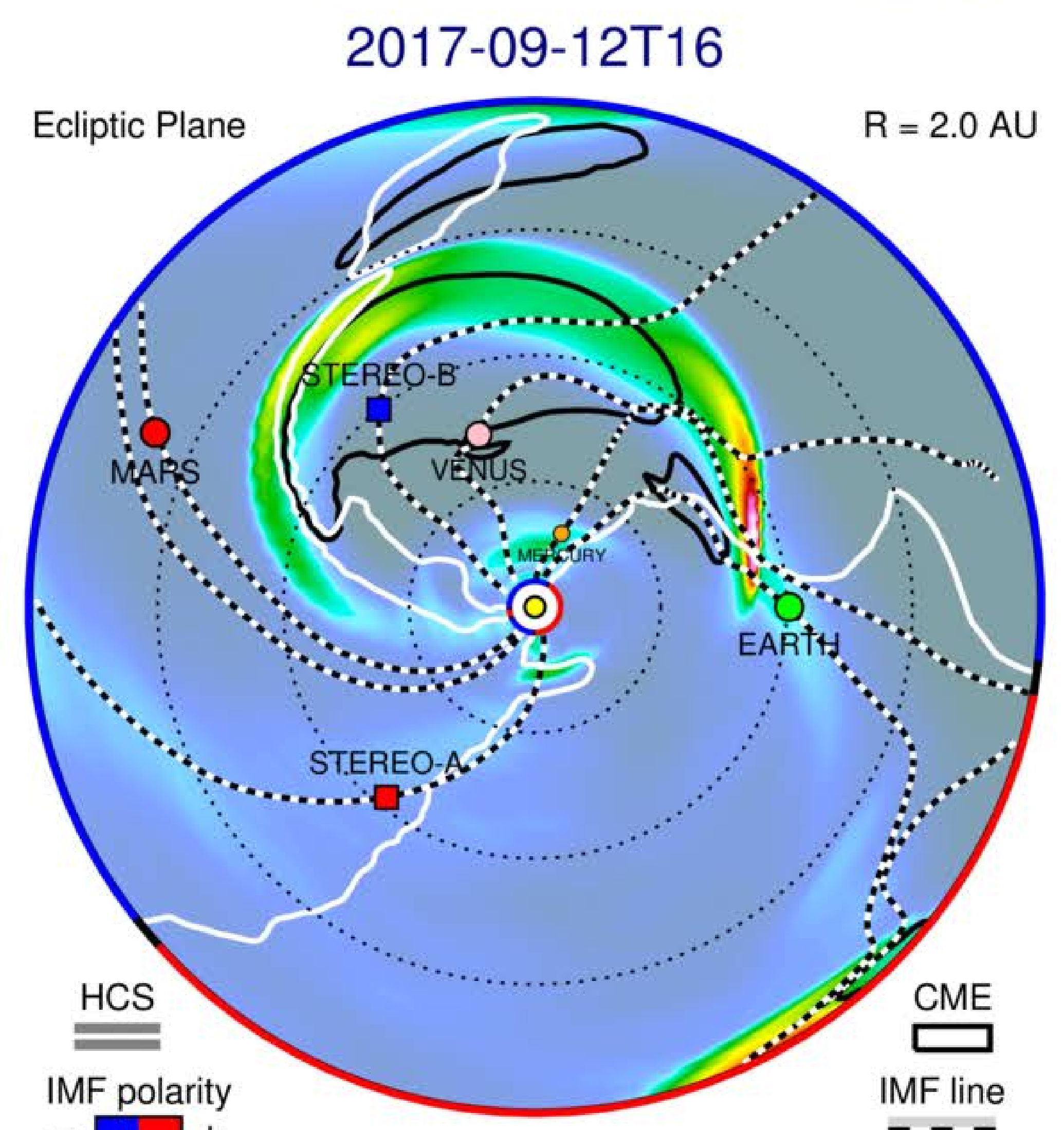

Normalized Solar Wind Number Density

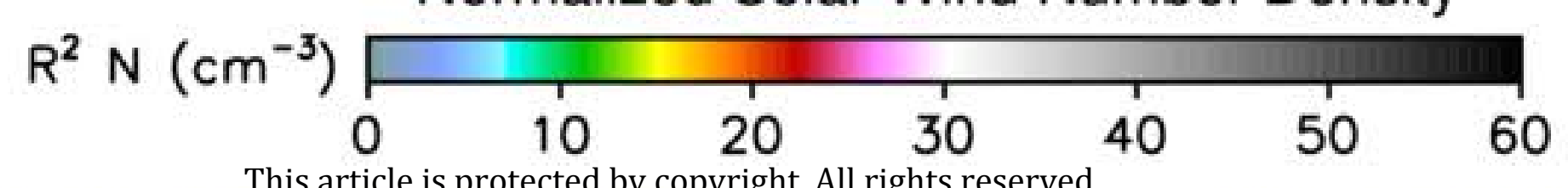
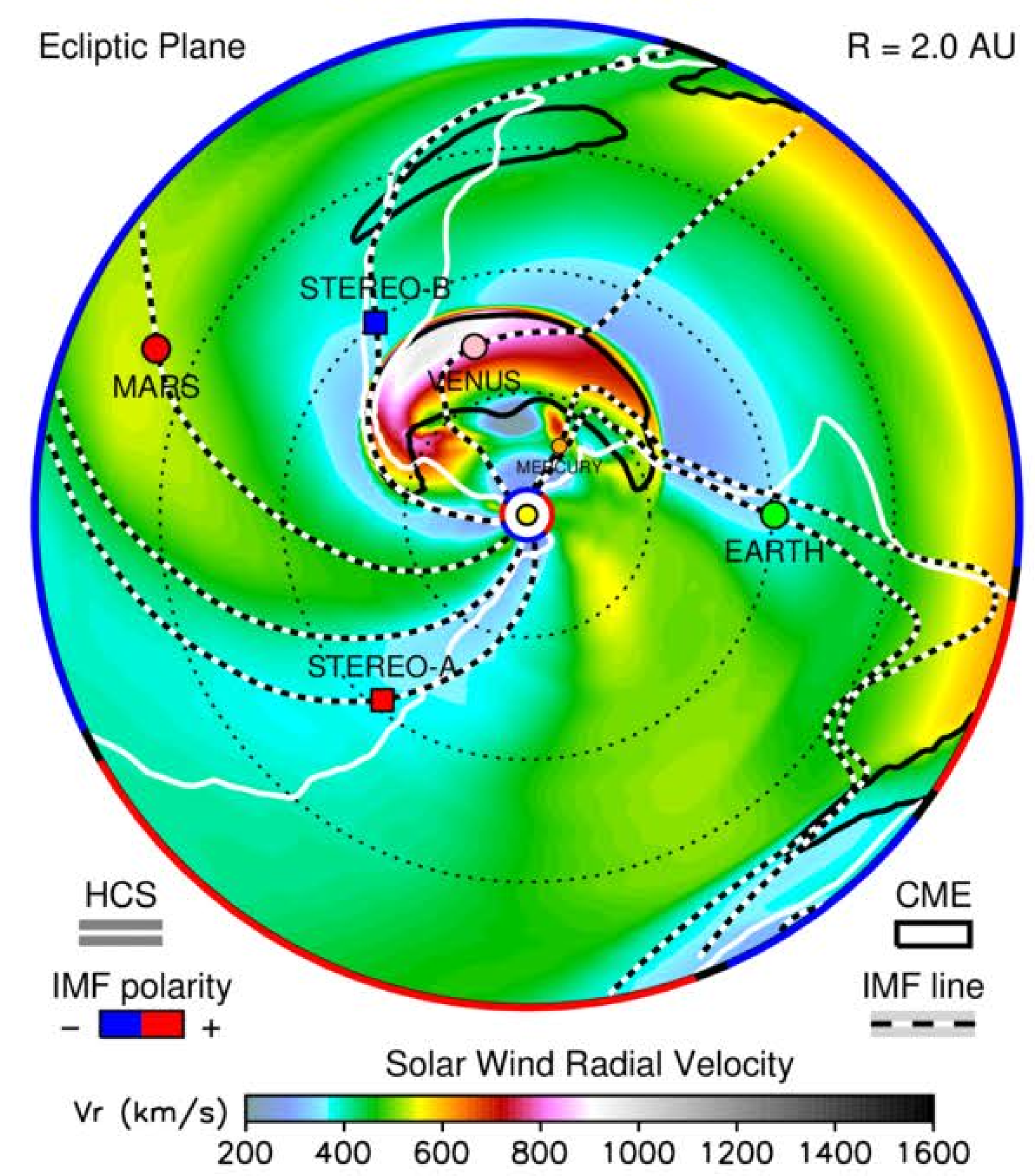

2017-09-12T16

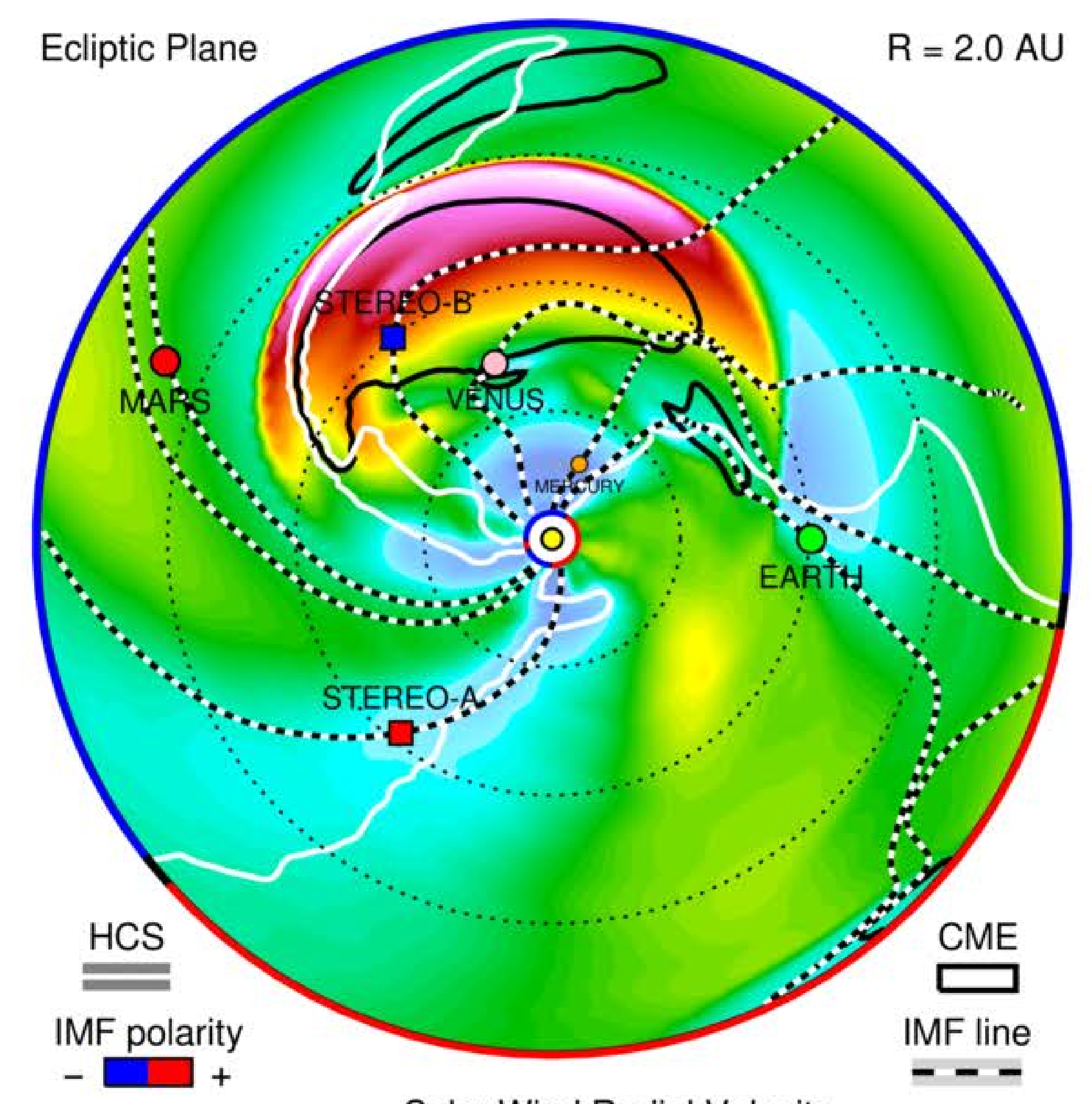

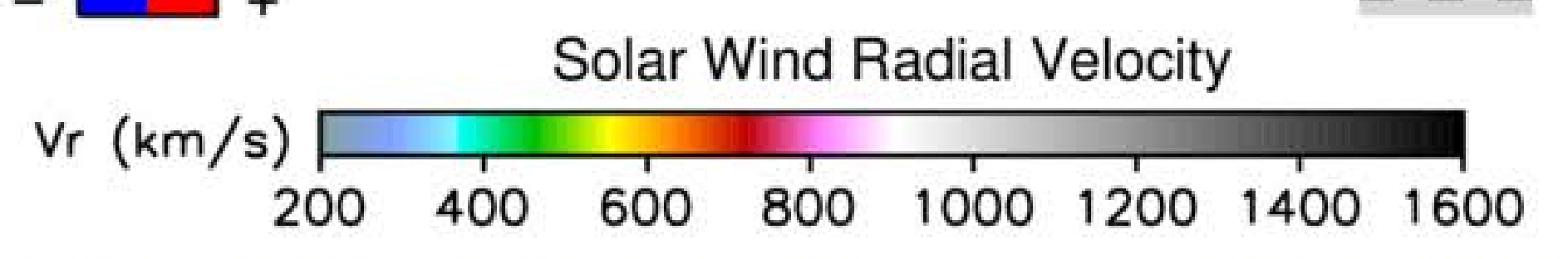

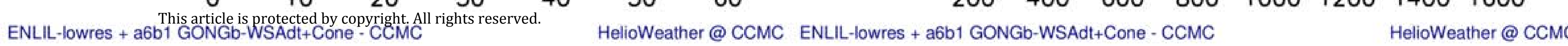




\section{Successive CMEs Causing Acceleration of}

\section{High Energy SEPs directed near the Earth \& Moon}

\section{9/6/2017 11:58 UT X9.3 9/10/2017 16:06 UT X8.2}

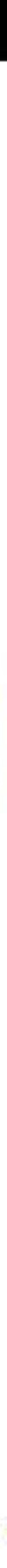

ICME 1

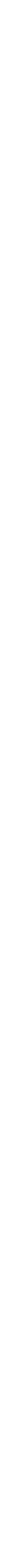




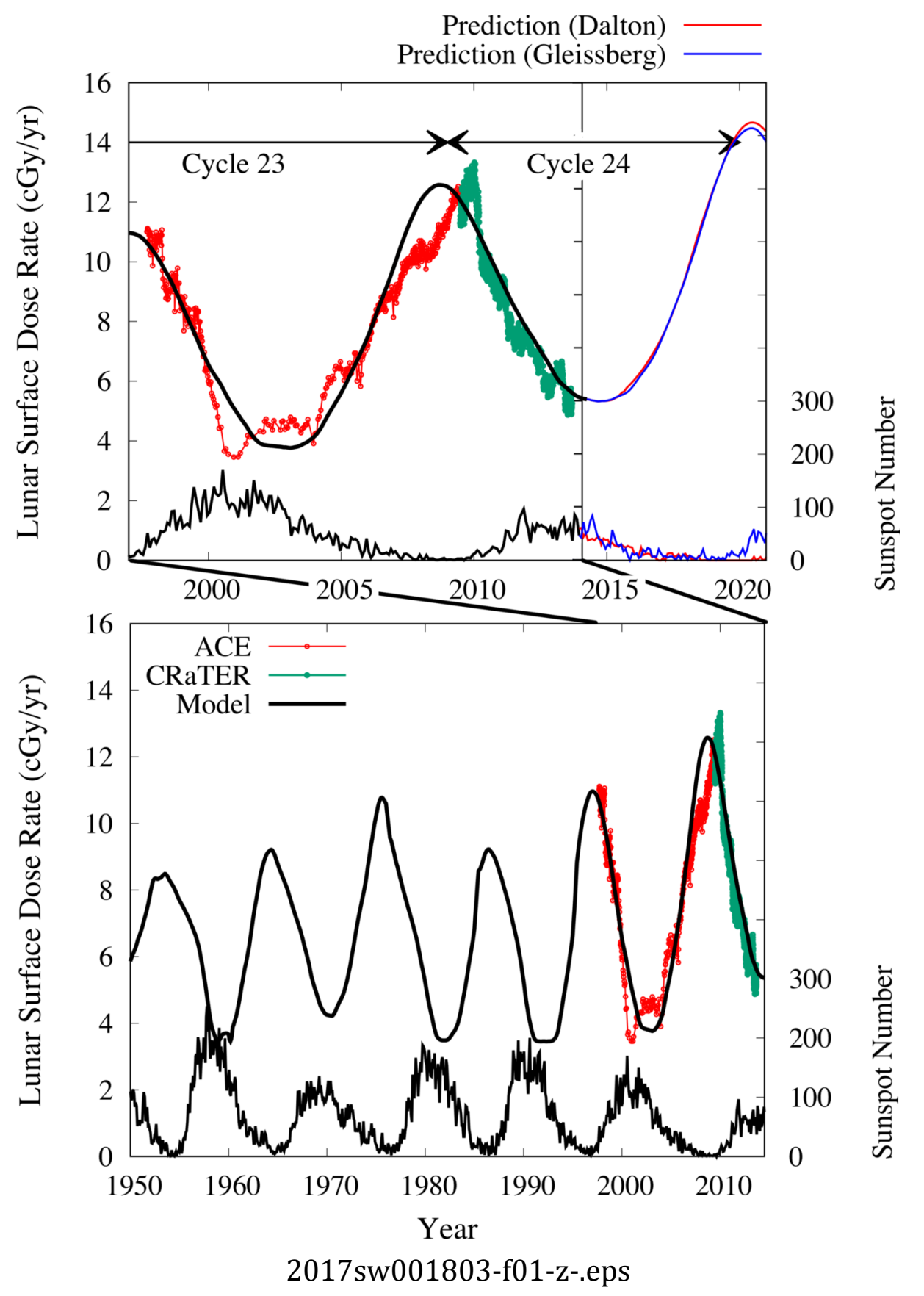

This article is protected by copyright. All rights reserved. 


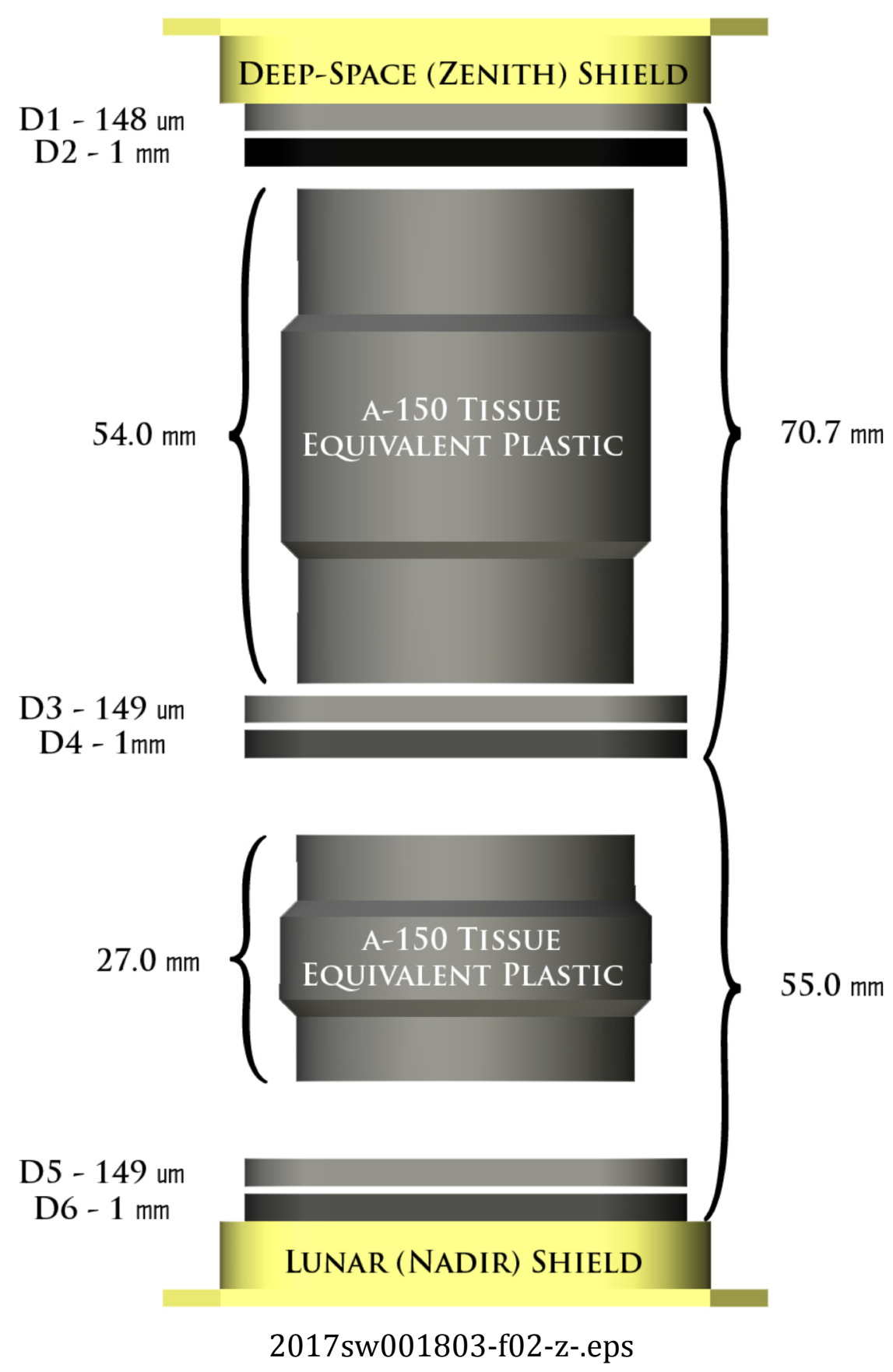

This article is protected by copyright. All rights reserved. 


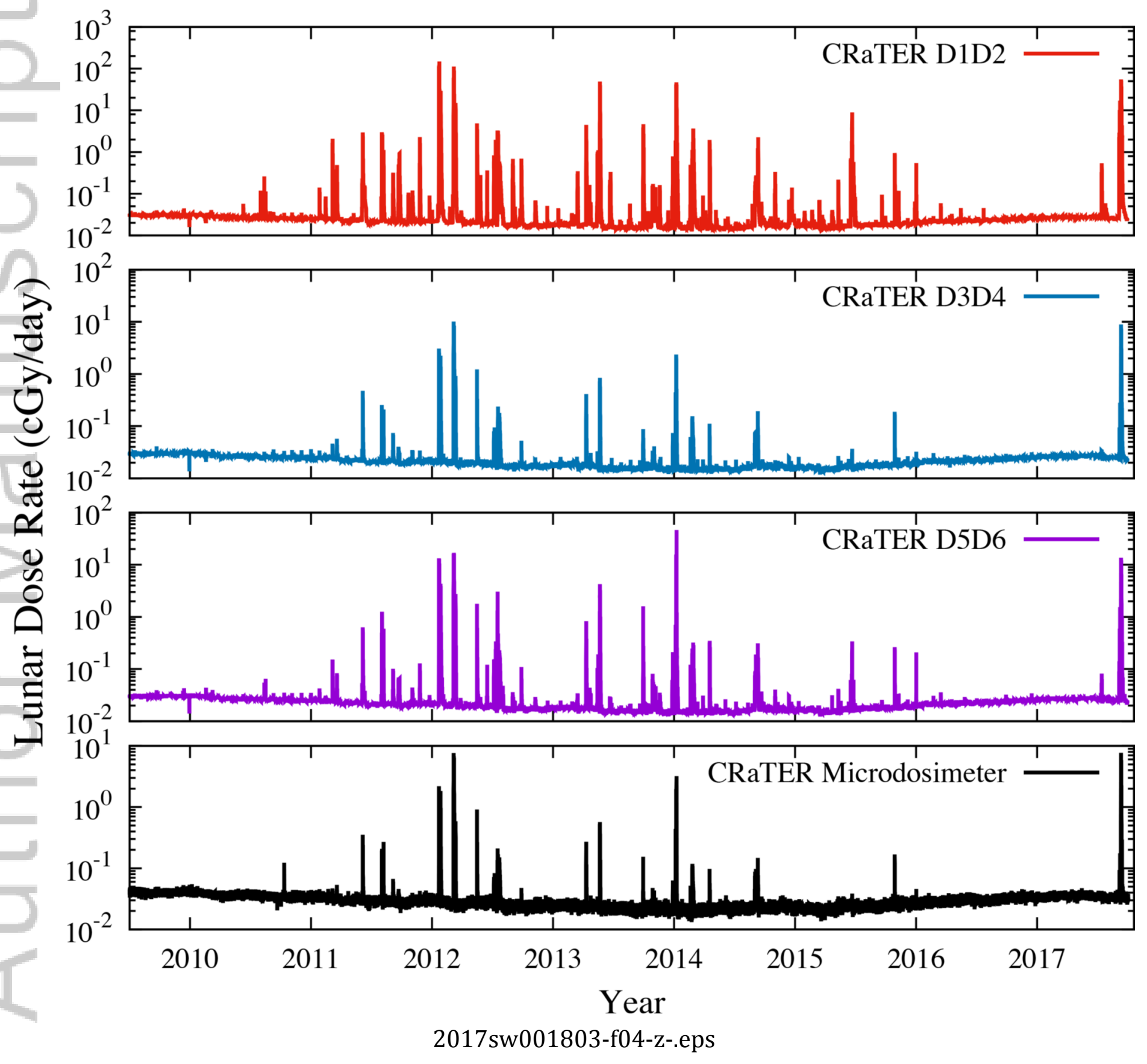

This article is protected by copyright. All rights reserved. 


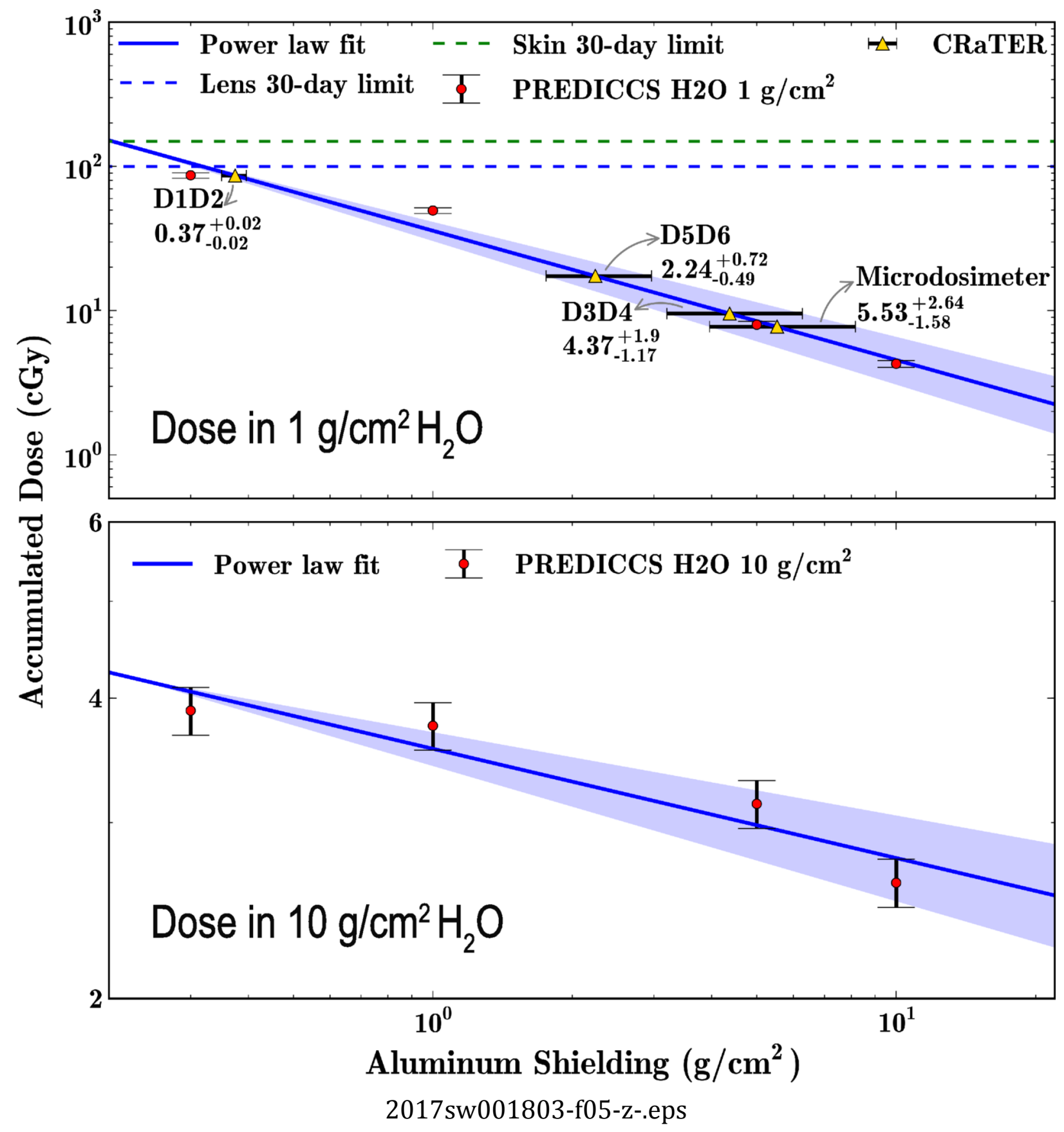

This article is protected by copyright. All rights reserved. 


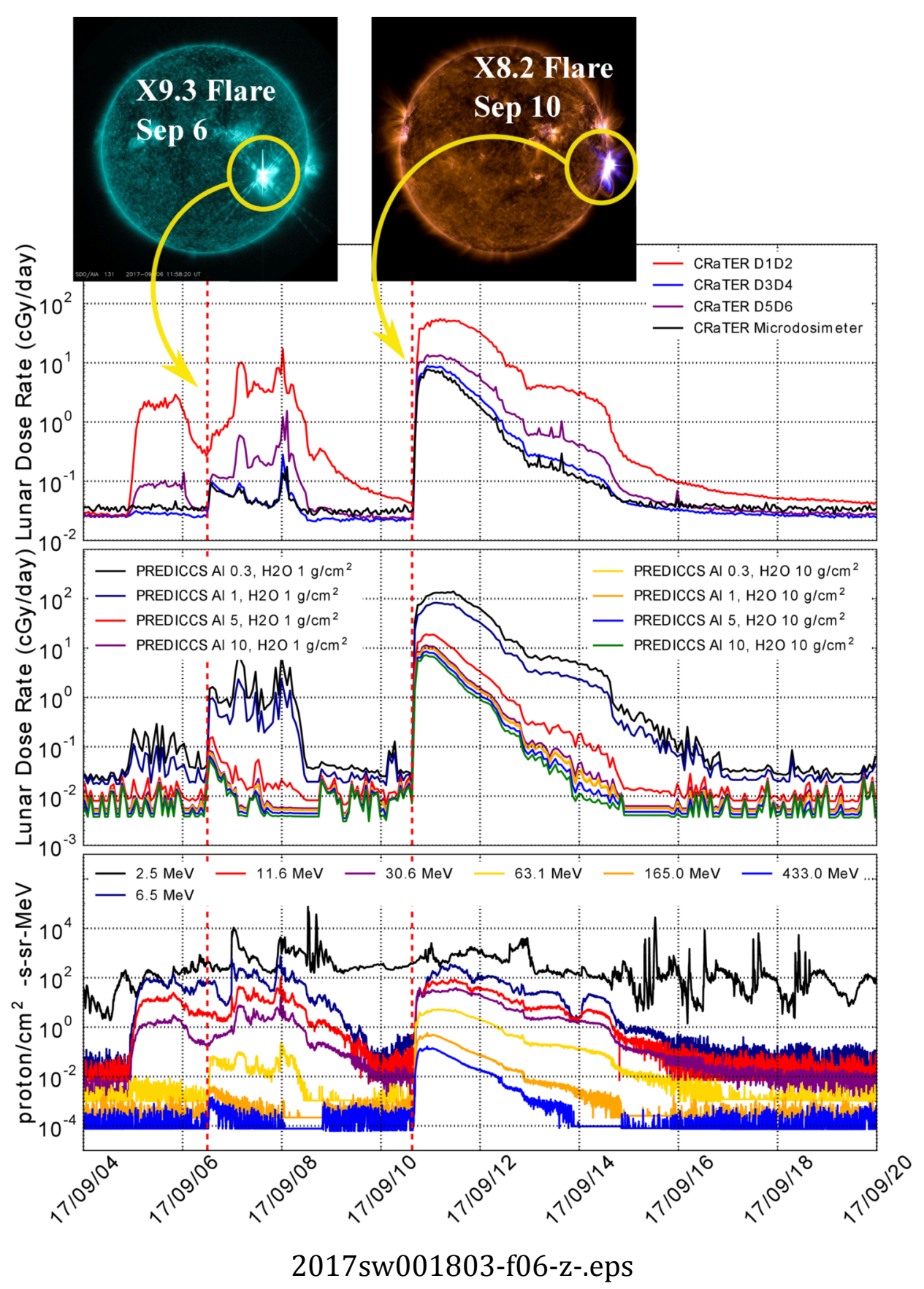

This article is protected by copyright. All rights reserved. 


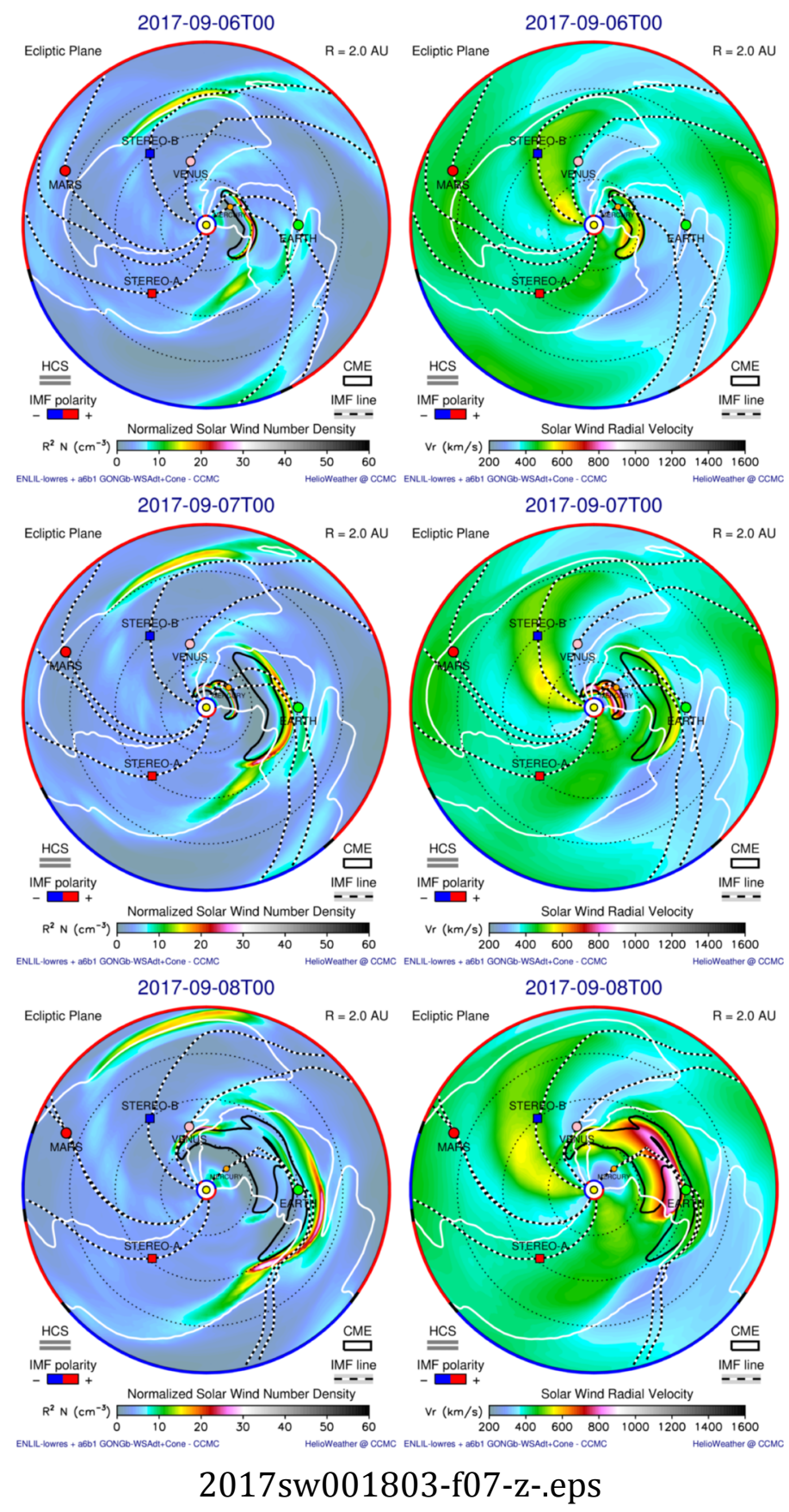

This article is protected by copyright. All rights reserved. 


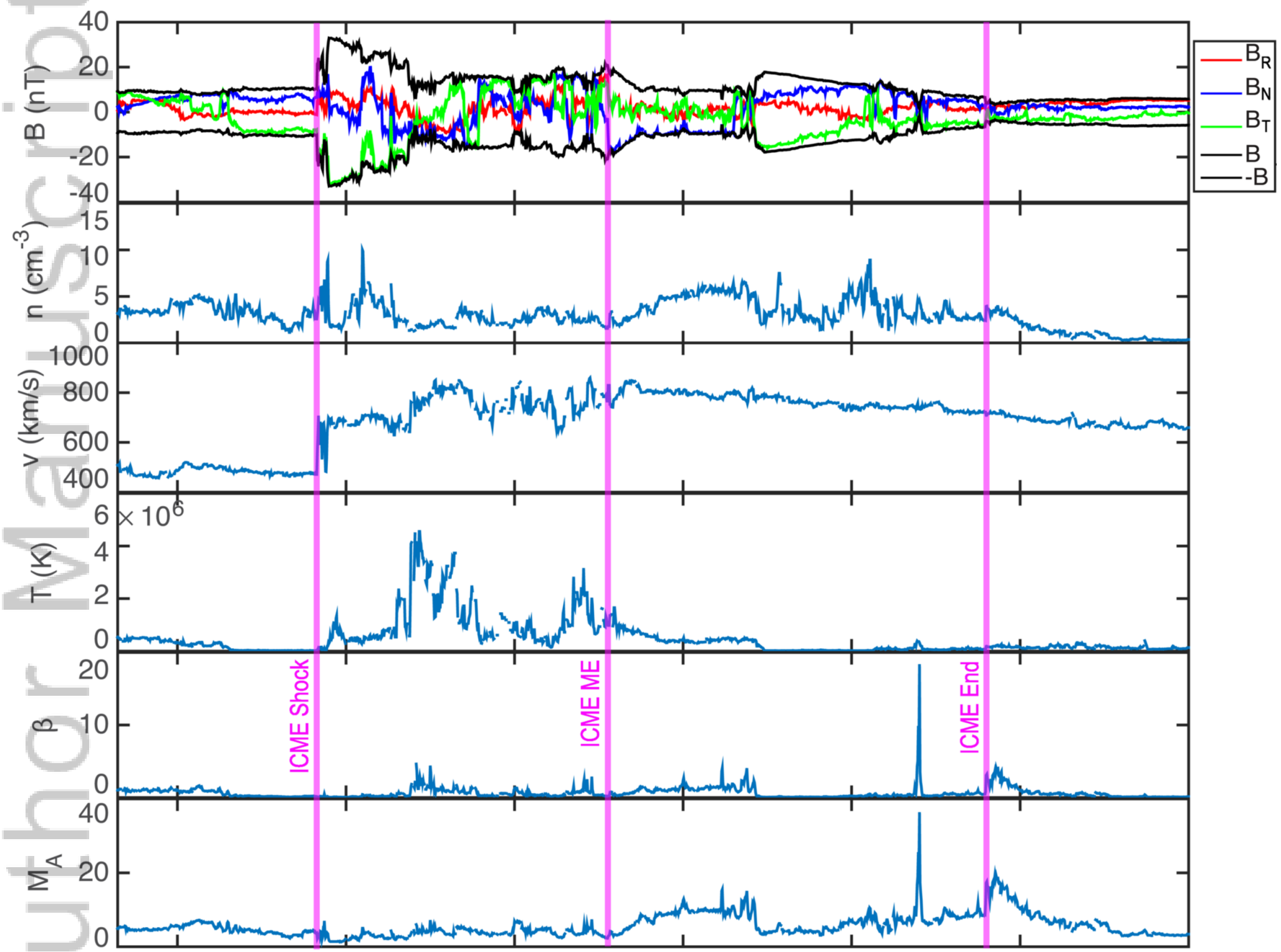

09/07 19:12 09/08 00:00 09/08 04:48 09/08 09:36 09/08 14:24 09/08 19:12 09/09 00:00 UT

2017sw001803-f08-z-.eps

This article is protected by copyright. All rights reserved. 


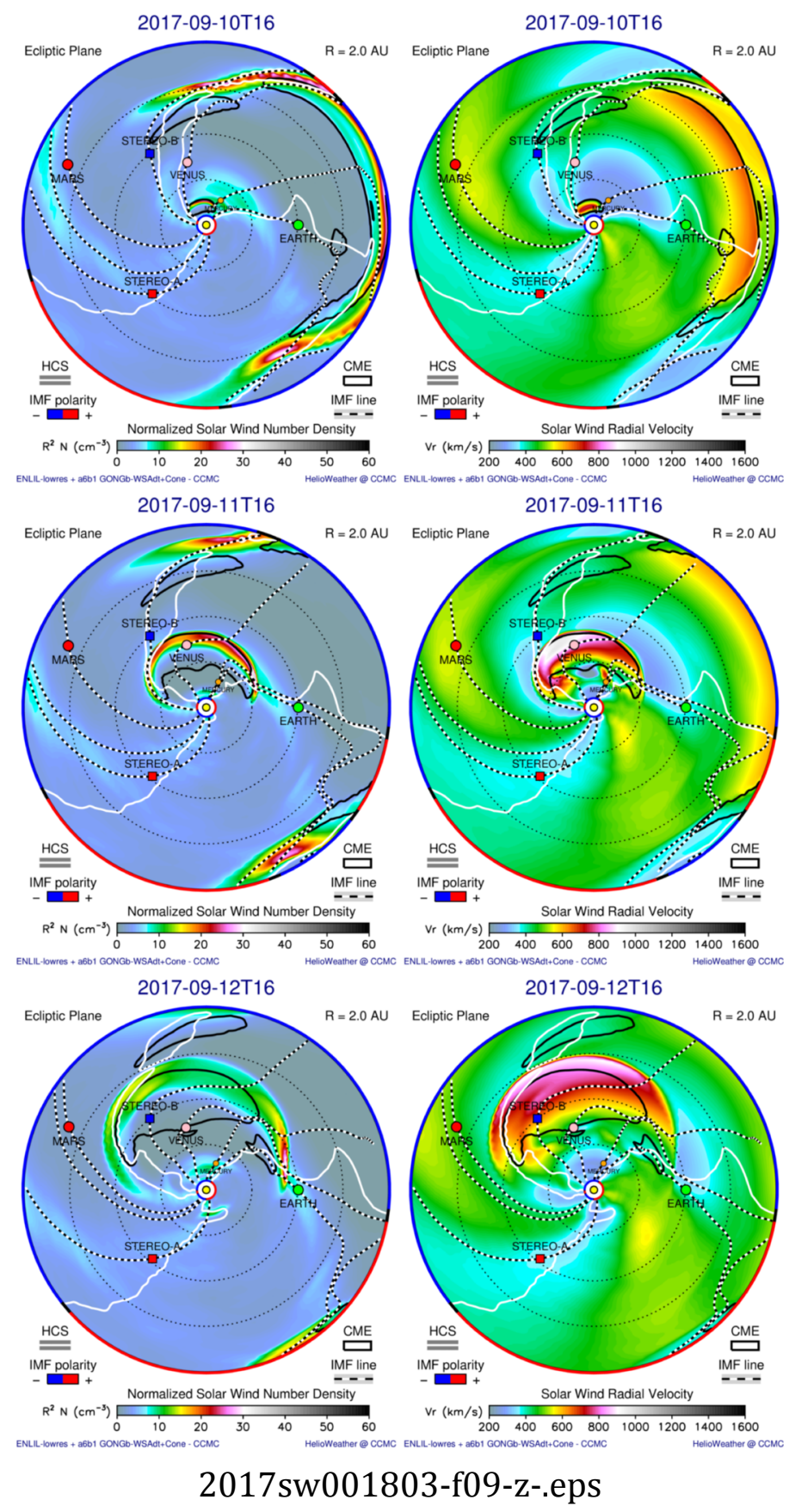

This article is protected by copyright. All rights reserved. 


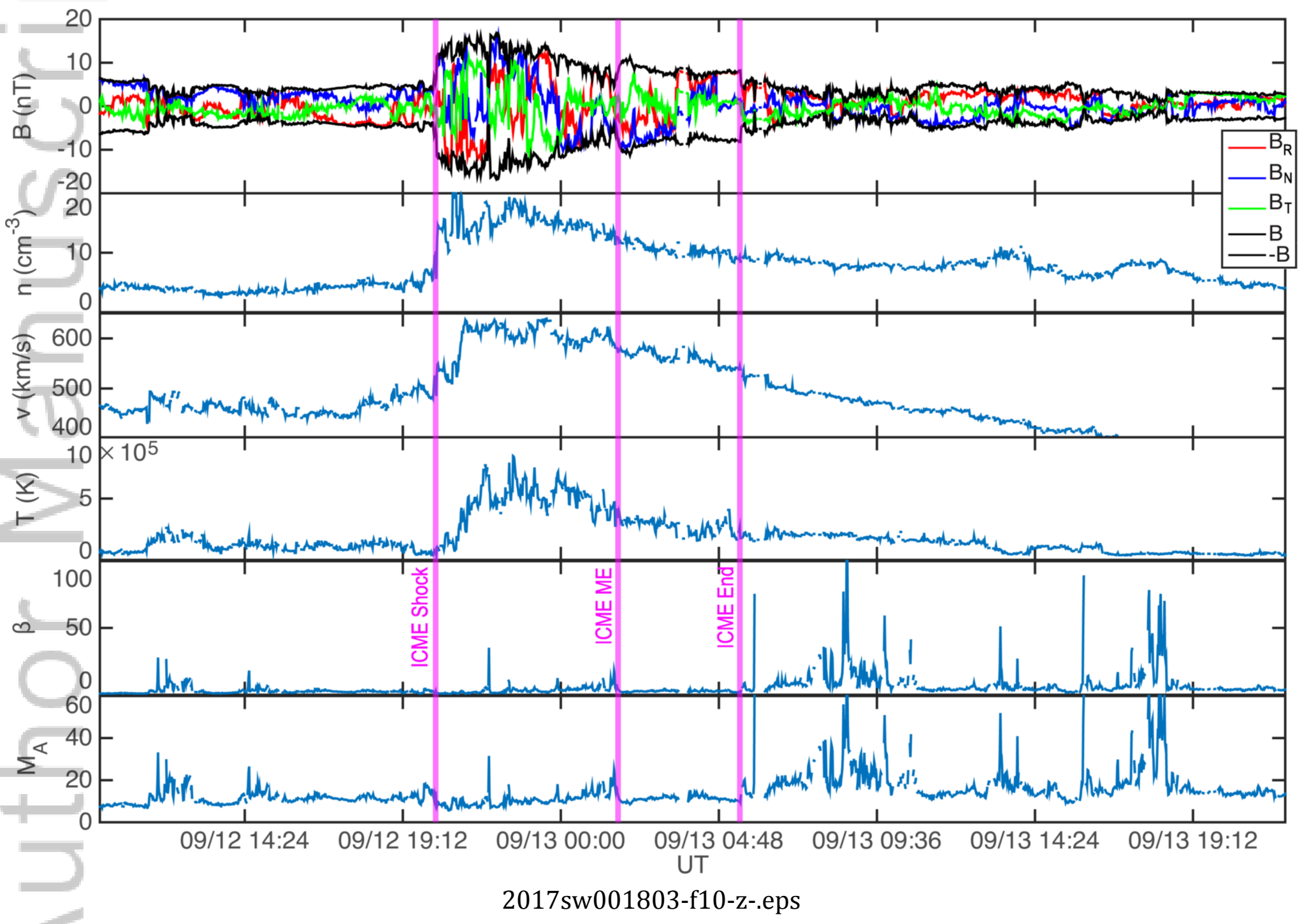

This article is protected by copyright. All rights reserved. 
Successive CMEs Causing Acceleration of High Energy SEPs directed near the Earth \& Moon
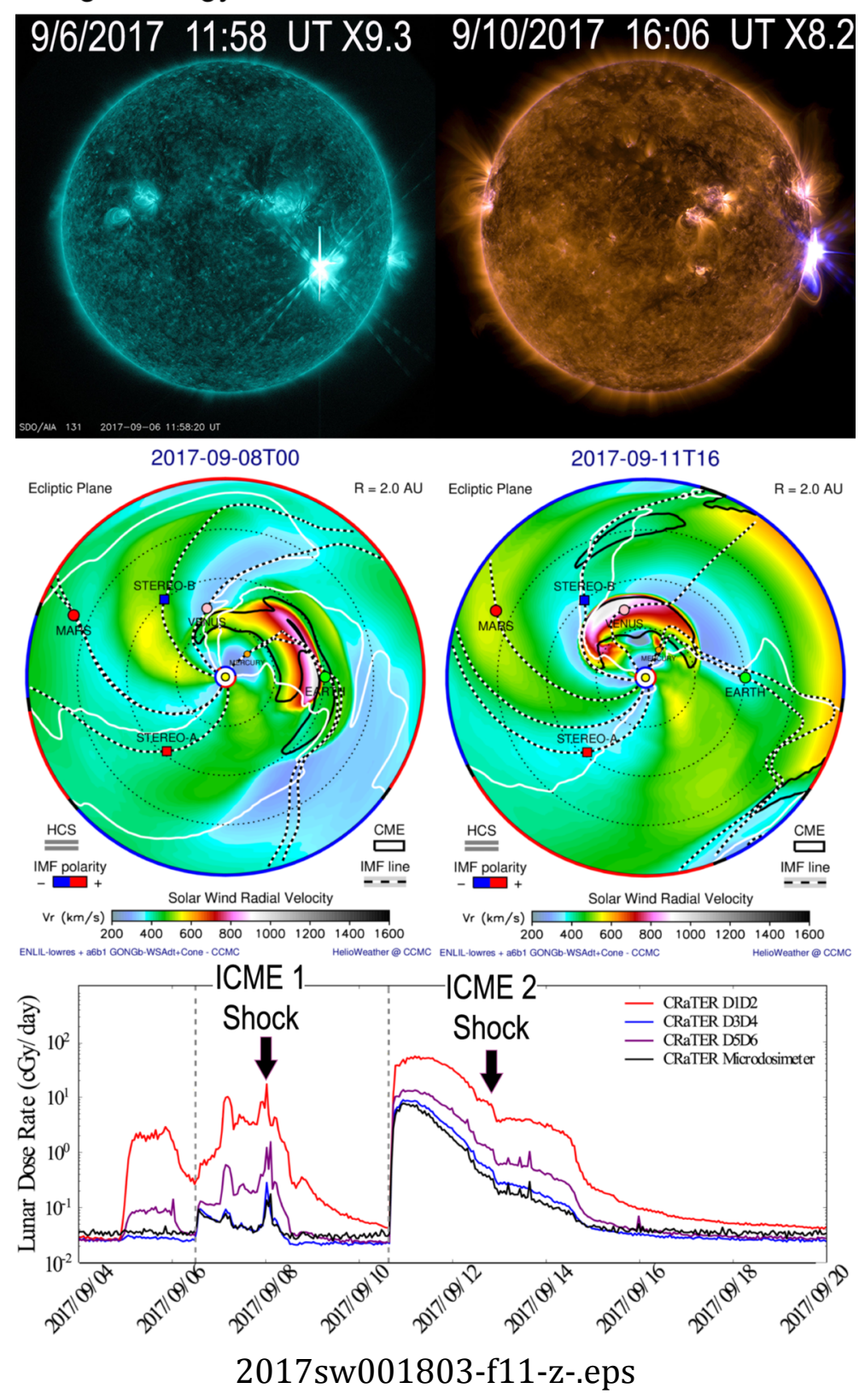

This article is protected by copyright. All rights reserved. 